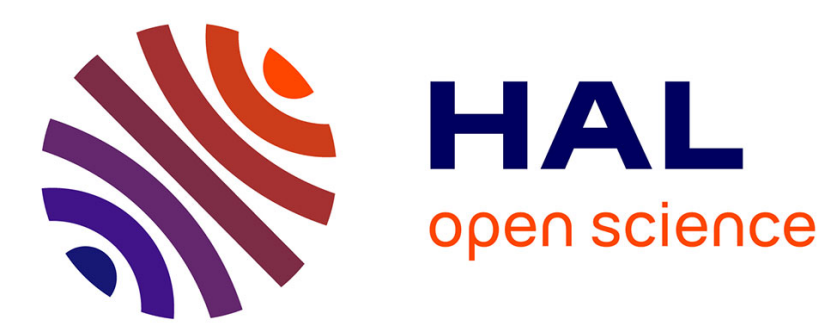

\title{
Some links between Cosserat, strain gradient crystal plasticity and the statistical theory of dislocations
}

Samuel Forest

\section{To cite this version:}

Samuel Forest. Some links between Cosserat, strain gradient crystal plasticity and the statistical theory of dislocations. Philosophical Magazine, 2009, 88, pp.3549-3563. 10.1080/14786430802154815 . hal-00513901

\section{HAL Id: hal-00513901 \\ https://hal.science/hal-00513901}

Submitted on 1 Sep 2010

HAL is a multi-disciplinary open access archive for the deposit and dissemination of scientific research documents, whether they are published or not. The documents may come from teaching and research institutions in France or abroad, or from public or private research centers.
L'archive ouverte pluridisciplinaire HAL, est destinée au dépôt et à la diffusion de documents scientifiques de niveau recherche, publiés ou non, émanant des établissements d'enseignement et de recherche français ou étrangers, des laboratoires publics ou privés. 


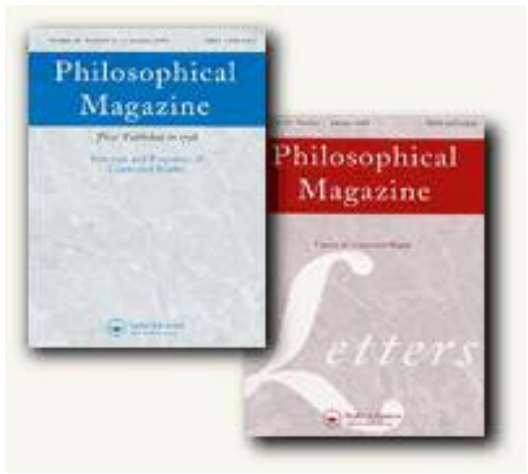

\section{Some links between Cosserat, strain gradient crystal plasticity and the statistical theory of dislocations}

\begin{tabular}{|c|c|}
\hline Journal: & Philosophical Magazine \& Philosophical Magazine Letters \\
\hline Manuscript ID: & TPHM-07-Dec-0375.R1 \\
\hline Journal Selection: & Philosophical Magazine \\
\hline $\begin{array}{l}\text { Date Submitted by the } \\
\text { Author: }\end{array}$ & 18-Apr-2008 \\
\hline Complete List of Authors: & $\begin{array}{l}\text { Forest, Samuel; Mines de Paris / CNRS / ParisTech, Centre des } \\
\text { Matériaux UMR } 7633\end{array}$ \\
\hline Keywords: & constitutive equations, plasticity of crystals \\
\hline Keywords (user supplied): & Cosserat, Gradient plasticity, Dislocation density \\
\hline \multicolumn{2}{|c|}{$\begin{array}{l}\text { Note: The following files were submitted by the author for peer review, but cannot be converted } \\
\text { to PDF. You must view these files (e.g. movies) online. }\end{array}$} \\
\hline $\begin{array}{l}\text { Forest-iutam07Eindhoven-re } \\
\text { Forest-iutam07Eindhoven-re } \\
\text { Forest-iutam07Eindhoven-re } \\
\text { Forest-iutam07Eindhoven-re } \\
\text { Forest-iutam07Eindhoven-re } \\
\text { lamacro.tex } \\
\text { tPHM2e.cls } \\
\text { upmath.sty }\end{array}$ & $\begin{array}{l}\text { /ised.aux } \\
\text { /ised.bbl } \\
\text { /ised.blg } \\
\text { /ised.log } \\
\text { ised.tex }\end{array}$ \\
\hline
\end{tabular}

\section{S) ScholaroNE \\ Manuscript Central}




\title{
Some links between Cosserat, strain gradient crystal plasticity and the statistical theory of dislocations
}

\author{
Samuel FOREST* \\ Mines Paris, ParisTech, CNRS UMR 7633 \\ BP 87, 91003 Evry Cedex, France \\ (revised)
}

\begin{abstract}
A link is established between a phenomenological Cosserat model of crystal plasticity due to [1] and recent results obtained in the statistical theory of dislocations by [2]. The existence of a back-stress related to the divergence of the couple stress tensor is derived. According to several dislocation-based models of single slip, the kinematic hardening modulus is found to be inversely proportional to dislocation density. Phenomenological extensions to multislip situations can be proposed based on these generalized continuum approaches.
\end{abstract}

\section{Introduction}

First links between the continuum theory of dislocations and the mechanics of generalized continua were established in the pioneering works of Kröner and Mura [3,4]. The connection between the dislocation density tensor and the rotational part of the plastic deformation was an incentive for the development of strain gradient crystal plasticity theories, based on the second gradient medium as proposed in [5]. On the other hand, the relation between the dislocation density tensor and lattice curvature derived by Nye [6] prompted several authors as recorded in [1] to formulate a Cosserat model for crystal plasticity. Since then a plethora of strain gradient plasticity models have been proposed without clear classification nor guidelines for assessing the fundamental differences between them. One of the objectives of this work is to show that the Cosserat theory and strain gradient plasticity models based on the introduction of the curl of plastic strain share major common features and belong to the same class of generalized crystal plasticity models.

Recent formulations of generalized continuum models incorporating the effect of the curl of plastic deformation within the framework of continuum thermomechanics show that this additional contribution to hardening compared to classical crystal plasticity, arises as a gradient-dependent internal stress or back-stress in the constitutive equations $[7,8,9]$.

In the mean time, the statistical theory of dislocations has made significant progress since the early attempts like [10]. In particular, higher order gradient terms in the continuum description have been derived in $[11,2]$ from spatial correlations of short-range dislocation-dislocation interactions, at least in the case of populations of straight parallel edge dislocations. This contribution was shown to be adequately represented by a back-stress component which is inversely proportional to the dislocation density. Closely related scaling laws were established for specific distributions of dislocations in relation to the Cosserat continuum theory $[3,12,13]$.

Comparisons with numerical simulations based on two-dimensional dislocation dynamics support the pertinence of this formulation for gradient-dependent internal stresses [14]. This contribution is essentially proportional to the second derivative of slip in the slip direction. The objective of the present work is to show that the mentioned Cosserat and strain gradient plasticity models lead to the same formulation of internal stresses, from purely phenomenological arguments, thus bridging a gap between statistical and constitutive theories, at least in the case of single slip.

${ }^{*}$ Corresponding author. Email: samuel.forest@ensmp.fr

Philosophical Magazine

ISSN 1478-6435 print/ISSN 1478-6443 online (c) 200x Taylor \& Francis

http://www.tandf.co.uk/journals

DOI: $\operatorname{xxxxxxxx}$ 
Extensions of the continuum model to multiple slip currently remain on a heuristic basis. Such extensions were recently proposed in $[15,16]$. The phenomenological constitutive approach making use of generalized continua also systematically provides such $3 \mathrm{D}$ formulations. It will be shown in the present work that the phenomenological and statistical approaches share several common features in the case of single slip. Accordingly, it is worth comparing the 3D formulations of both classes of models. Such gradient dependent internal stresses can be embedded in more comprehensive and realistic constitutive models for the deformation of metals and alloys, as shown recently in [17].

We start to recall the Cosserat crystal plasticity framework focusing on the relation between the Cosserat microrotation and crystal lattice rotation. In particular, one additional elastic parameter of the Cosserat model will be interpreted as a Lagrange multiplier to make both rotations coincide. A Schmid law is then formulated in section 4 based on the generally non-symmetric Cosserat force stress tensor. It is shown to lead to the existence of a back-stress expressed in terms of couple stresses. The found internal stresses are explicited in the case of single slip in section 5 and compared to results from statistical description of dislocation behaviour in section 8 .

The derivations of the generalized continuum models are presented within the small deformation framework for the sake of simplicity. Extensions to finite deformation formulations are straightforward following the lines of $[1,18,19,20]$.

In this work, zeroth, first, second, third and fourth order tensors are denoted by $a, \underset{\boldsymbol{a}}{\boldsymbol{a}}, \underset{\sim}{\boldsymbol{a}}, \underset{\sim}{\boldsymbol{a}}, \boldsymbol{a}$ respectively. The simple and double contractions are written ., $:$. In index form with respect to an orthonormal Cartesian basis, these notations correspond to $\underline{\boldsymbol{a}} \cdot \underline{\boldsymbol{b}}=a_{i} b_{i}, \quad \underset{\sim}{\boldsymbol{a}}: \underset{\sim}{\boldsymbol{b}}=a_{i j} b_{i j}$, where repeated indices are summed up. The tensor product is denoted by $\otimes$. The nabla operator is denoted by $\boldsymbol{\nabla}$. For example, the component $i j k$ of $\boldsymbol{\nabla} \underset{\sim}{\boldsymbol{A}}$ is $A_{i j, k}$. For full clarity, both intrinsic and index notations are given at several places in the text.

\section{Cosserat crystal plasticity at small deformation}

The degrees of freedom of the theory are the displacement vector $\underline{\boldsymbol{u}}$ and the rotation pseudo-vector $\underline{\boldsymbol{\Phi}}$ which is associated with the skew-symmetric part of the micro-rotation tensor $\underset{\sim}{\boldsymbol{R}}$ in the case of small rotations:

$$
\underset{\sim}{\boldsymbol{R}} \simeq \underset{\sim}{\mathbf{1}}-\underline{\boldsymbol{\epsilon}} \cdot \underline{\boldsymbol{\Phi}}, \quad R_{i j} \simeq \delta_{i j}-\epsilon_{i j k} \Phi_{k}
$$

where $\epsilon_{i j k}$ denotes the permutation tensor. The deformation measures of the Cosserat theory are the relative deformation tensor $\underset{\sim}{\boldsymbol{e}}$ and the torsion-curvature tensor $\underset{\sim}{\boldsymbol{\kappa}}$ :

$$
\begin{gathered}
\underset{\sim}{e}=\nabla \underline{\boldsymbol{u}}+\underset{\sim}{\boldsymbol{\epsilon}} \underline{\Phi}, \quad \underset{\sim}{\boldsymbol{\kappa}}=\nabla \underline{\boldsymbol{\Phi}} \\
e_{i j}=u_{i, j}+\epsilon_{i j k} \Phi_{k}, \quad \kappa_{i j}=\Phi_{i, j}
\end{gathered}
$$

The stress measures associated with the deformation rates in the power of internal forces are the force stress tensor $\underset{\sim}{\boldsymbol{\sigma}}$ and the couple stress tensor $\underset{\sim}{\boldsymbol{m}}$. They are generally not symmetric. They must satisfy the balance equations for momentum and balance of momentum, written in the static case:

$$
\operatorname{div} \underset{\sim}{\boldsymbol{\sigma}}=0, \quad \sigma_{i j, j}=0
$$

$$
\operatorname{div} \underset{\sim}{\boldsymbol{m}}+2 \stackrel{\stackrel{\times}{\boldsymbol{\sigma}}}{\underline{\sim}}=0, \quad m_{i j, j}-\epsilon_{i k l} \sigma_{k l}=0
$$


where volume forces and couples are excluded for brevity. The axial vector associated with the skewsymmetric part of the stress tensor is denoted by:

$$
\stackrel{\times}{\underline{\boldsymbol{\sigma}}}=-\frac{1}{2} \underset{\underline{\epsilon}}{\boldsymbol{\epsilon}}: \underset{\sim}{\boldsymbol{\sigma}}, \quad \stackrel{\times}{\sigma}_{i}=-\frac{1}{2} \epsilon_{i k l} \sigma_{k l}
$$

It couples the balance of momentum and moment of momentum equations. Exponents ${ }^{s}$ and ${ }^{a}$ are introduced to respectively denote the symmetric and skew-symmetric parts of the corresponding tensor:

$$
\begin{gathered}
\underset{\sim}{\boldsymbol{\sigma}}={\underset{\sim}{\boldsymbol{\sigma}}}^{s}+{\underset{\sim}{\boldsymbol{\sigma}}}^{a}, \quad \stackrel{\boldsymbol{\sigma}}{a}^{a}=-\underset{\sim}{\boldsymbol{\epsilon}} \cdot \stackrel{\stackrel{\times}{\boldsymbol{\sigma}}}{=} \frac{1}{2} \underset{\sim}{\boldsymbol{\epsilon}} \cdot \underset{\underline{\epsilon}}{\boldsymbol{\epsilon}}: \underset{\sim}{\boldsymbol{\sigma}} \\
\sigma_{i j}^{a}=-\frac{1}{2} \epsilon_{i j k} \stackrel{\times}{\sigma}_{k}=\frac{1}{2} \epsilon_{i j k} \epsilon_{k m n} \sigma_{m n}
\end{gathered}
$$

The traction vector $\underline{\boldsymbol{t}}$ and couple stress vector $\underline{\boldsymbol{m}}$ acting on a surface element must fulfill the following boundary conditions:

$$
\begin{gathered}
\underline{\boldsymbol{t}}=\underset{\sim}{\boldsymbol{\sigma}} \cdot \underline{\boldsymbol{n}}, \quad t_{i}=\sigma_{i j} n_{j} \\
\underline{\boldsymbol{m}}=\underset{\sim}{\boldsymbol{m}} \underline{\underline{\boldsymbol{n}}}, \quad m_{i}=m_{i j} n_{j}
\end{gathered}
$$

where $\underline{\boldsymbol{n}}$ is the outward oriented normal vector at any point of the boundary of the body. The relative deformation can be split into elastic and plastic parts,

$$
\underset{\sim}{e}={\underset{\sim}{e}}^{e}+\underline{\sim}^{p}, \quad e_{i j}=e_{i j}^{e}+e_{i j}^{p}
$$

Such a decomposition is not introduced in this work for the total curvature $\underset{\sim}{\boldsymbol{\sim}}$ for the sake of simplicity. A partition of curvature was considered in $[1,21]$ and it is not recalled here. The elastic deformation and the total curvature tensors are assumed to be linked with the force and couple stress tensors, respectively, by a generalized Hooke law:

$$
\begin{gathered}
\underset{\sim}{\boldsymbol{\sigma}}=\underset{\sim}{\boldsymbol{E}}:{\underset{\sim}{e}}^{e}, \quad \sigma_{i j}=E_{i j k l} e_{k l}^{e} \\
\underset{\sim}{\boldsymbol{m}}=\underset{\sim}{\boldsymbol{C}}: \underset{\sim}{\boldsymbol{\kappa}}, \quad m_{i j}=C_{i j k l} \kappa_{k l}
\end{gathered}
$$

where $\underset{\sim}{\boldsymbol{E}}$ is the fourth order tensor of elastic moduli (unit $\mathrm{MPa}$ ) and $\underset{\approx}{\boldsymbol{C}}$ are secant moduli of torsion and bending stiffness (unit MPa.mm²). In the isotropic case, these tensors are built from 6 independent elastic moduli:

$$
\begin{gathered}
\underset{\sim}{\boldsymbol{\sigma}}=\lambda\left(\operatorname{trace}{\underset{\sim}{\boldsymbol{e}}}^{e}\right) \underset{\sim}{\mathbf{1}}+2 \mu{\underset{\sim}{\boldsymbol{e}}}^{e s}+2 \mu_{c} \stackrel{\sim}{\boldsymbol{e}}^{e a} \\
\underset{\sim}{\boldsymbol{m}}=\alpha(\operatorname{trace} \underset{\sim}{\boldsymbol{\kappa}}) \underset{\sim}{\mathbf{1}}+2 \beta{\underset{\sim}{\boldsymbol{\kappa}}}^{s}+2 \gamma \underset{\sim}{\boldsymbol{\kappa}}
\end{gathered}
$$

The Lamé constants are $\lambda$ and $\mu$. The coupling modulus $\mu_{c}$ relates the skew-symmetric part of the relative deformation tensor to the skew-symmetric part of the stress tensor. The additional Cosserat parameters $\alpha, \beta, \gamma$ are intrinsic torsion and bending stiffnesses. We adopt the simplification $\beta=\gamma$ as in [22]. 
The kinematics of plastic flow is dictated by the orientation tensors $\underset{\sim}{\boldsymbol{P}}{ }^{s}$ associated with the crystallography of the $N$ slip systems:

$$
\stackrel{\dot{e}}{p}^{p}=\sum_{\alpha=1}^{N} \dot{\gamma}^{\alpha}{\underset{\sim}{\boldsymbol{P}}}^{\alpha}, \quad \text { with } \quad{\underset{\sim}{\boldsymbol{P}}}^{\alpha}=\underline{\boldsymbol{l}}^{\alpha} \otimes \underline{\boldsymbol{n}}^{\alpha}
$$

where $\underline{\boldsymbol{l}}^{\alpha}$ and $\underline{\boldsymbol{n}}^{\alpha}$ respectively are the slip direction and the normal to the slip plane vectors for slip system number $\alpha$. For each slip system, the increment of plastic slip is $\dot{\gamma}^{\alpha}$.

\section{Cosserat microrotation and lattice rotation}

The lattice rotation rate $\boldsymbol{w}^{e}$ is defined as the difference between the material rotation rate $\underset{\sim}{\boldsymbol{w}}$ and the plastic rotation rate represented by the skew-symmetric part of plastic deformation [23]:

$$
\begin{aligned}
& \underline{\sim}^{e}=(\boldsymbol{\nabla} \underline{\dot{u}})^{a}-\underline{\sim}^{p a} \\
& =\stackrel{\sim}{\dot{e}}^{a}-\underset{\sim}{\boldsymbol{\epsilon}} . \underline{\dot{\Phi}}-\stackrel{\dot{e}}{p a}^{p a} \\
& =\stackrel{\dot{e}}{e}^{e a}-\underset{\sim}{\epsilon} \cdot \underline{\dot{\Phi}}
\end{aligned}
$$

which provides a relation between the lattice rotation rate ${\underset{\sim}{\boldsymbol{w}}}^{e}$ and the Cosserat microrotation rate $-\underset{\sim}{\boldsymbol{\epsilon}} . \underline{\dot{\Phi}}$. Accordingly, the Cosserat microrotation can be identified with lattice rotation if and only if the following internal constraint is enforced:

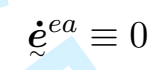

that is when the skew-symmetric part of the elastic relative deformation vanishes. In the works [21, 13], this constraint was enforced by a penalty method which consists in setting a high enough value of the constitutive parameter $\mu_{c}$. This parameter could also be treated as a Lagrange multiplier to ensure the identification between Cosserat rotation and lattice rotation. In that case, the skew-symmetric part of the force stress tensor must be regarded as a reaction force associated with the internal constraint. A similar (but different) situation is met in the so-called Koiter or couple stress theory for which the Cosserat microrotation coincides with the material rotation itself [24].

When the constraint (20) is enforced, the Cosserat directors are lattice vectors. In that case, the following relationship is obtained between Cosserat, material and lattice rotations:

$$
-\underline{\boldsymbol{\epsilon}} \cdot \underline{\dot{\Phi}}=\boldsymbol{w}^{e}=\underset{\sim}{\boldsymbol{w}}-\boldsymbol{w}^{p}
$$

where $\underset{\sim}{\boldsymbol{w}}=(\boldsymbol{\nabla} \underline{\dot{\boldsymbol{u}}})^{a}, \boldsymbol{\sim}^{p}={\underset{\sim}{\dot{e}}}^{p a}$ are the skew-symmetric parts of the total and plastic deformation rates respectively. The associated rotation vectors therefore satisfy the relation:

$$
\underline{\dot{\Phi}}=\underline{x}^{\stackrel{\times}{\boldsymbol{w}}} \underline{\underline{w}}^{p}
$$

\section{Schmid law with a non-symmetric stress tensor}

Plastic slip is activated when the resolved shear stress on a given slip system reaches a critical value. The resolved shear stress $\tau^{s}$ is the component of the traction vector acting on the slip plane in the slip direction:

$$
\begin{aligned}
\tau^{\alpha} & =\left(\underset{\sim}{\boldsymbol{\sigma}} \cdot \underline{\boldsymbol{n}}^{\alpha}\right) \cdot \underline{\boldsymbol{l}}^{\alpha}=\underset{\sim}{\boldsymbol{\sigma}}:{\underset{\sim}{\boldsymbol{P}}}^{\alpha}=\boldsymbol{\sigma}^{s}:{\underset{\sim}{\boldsymbol{P}}}^{\alpha s}+{\underset{\sim}{\boldsymbol{\sigma}}}^{a}:{\underset{\sim}{\boldsymbol{P}}}^{\alpha a} \\
& =\tau^{\mathrm{sym} \alpha}-x^{\alpha}
\end{aligned}
$$


A decomposition of the total resolved shear stress is found into a component $\tau^{\text {sym }}$ which is nothing but the resolved shear stress computed with the symmetrized stress tensor, and an internal stress variable $x^{s}$ defined as:

$$
x^{\alpha}=-\underline{\sim}^{a}:{\underset{\sim}{\boldsymbol{P}}}^{\alpha a}=\underline{\boldsymbol{\sigma}}^{\times} \cdot\left(\underline{\boldsymbol{l}}^{\alpha} \wedge \underline{\boldsymbol{n}}^{\alpha}\right)
$$

where $\wedge$ denotes the vector product. The additional contribution associated with the skew-symmetric part of the stress will therefore act as a back-stress in the yield criterion. An alternative expression of the internal stress can be worked out by taking the balance of moment of momentum equation (5) into account:

$$
x^{\alpha}=-\frac{1}{2}(\operatorname{div} \underset{\sim}{\boldsymbol{m}}) \cdot\left(\underline{\boldsymbol{l}}^{\alpha} \wedge \underline{\boldsymbol{n}}^{\alpha}\right)
$$

which relates the internal stress $x^{\alpha}$ to a projection of the divergence of the couple stress tensor.

The slip activation criterion for slip system $\alpha$ is

$$
f^{\alpha}\left(\boldsymbol{\sigma}, \tau_{c}^{\alpha}\right)=\left|\tau^{\alpha}\right|-\tau_{c}^{\alpha}=\left|\tau^{\mathrm{sym} \alpha}-x^{s}\right|-\tau_{c}^{\alpha}=0
$$

This criterion involves the critical resolved shear stress $\tau_{c}^{\alpha}$ for which usual hardening laws of crystal plasticity can be used. At this stage, the evolution of $\tau_{c}^{\alpha}$ can be limited to the effect of so-called statistically stored dislocations (density $\rho^{S}$ ). The effect of the dislocation density tensor, or equivalently so-called geometrically necessary dislocation densities (GND), enters the model via the back-stress $x^{s}$ related to couple stresses and therefore to lattice curvature, as a result of the celebrated Nye relation connecting the dislocation density tensor and lattice curvature [6]. This is at variance with the quite common modification of Taylor's rule, namely $\tau_{c}$ proportional to $\sqrt{\rho^{S}+\rho^{G}}$, where different dislocation density measures are combined in the friction stress [25].

A viscoplastic flow rule is adopted to compute the amount of plastic glide:

$$
\dot{\gamma}=\left\langle\frac{f}{K}\right\rangle^{n} \operatorname{sign}\left(\tau^{\alpha}\right)
$$

in the form of a power law involving the viscosity parameter $K$ and the power $n$. The brackets denote the positive part of the quantity.

\section{$5 \quad$ Application to single slip}

We derive here the special form of the previous constitutive and balance equations when plastic deformation proceeds through single slip in a plane of normal $\underline{\boldsymbol{n}}$ in the direction $\underline{\boldsymbol{l}}$ :

$$
\stackrel{\sim}{\dot{e}}^{p}=\dot{\gamma} \underline{\boldsymbol{l}} \otimes \underline{\boldsymbol{n}}
$$

We adopt a Cartesian coordinate system such that the third vector of the basis is

$$
\underline{\boldsymbol{e}}_{3}=\underline{\boldsymbol{l}} \wedge \underline{\boldsymbol{n}}
$$

We assume that no gradient will develop along the out of plane direction. In this two-dimensional situation, the lattice rotation will take place with respect to the third axis:

$$
\underline{\boldsymbol{\Phi}}=\Phi_{3} \underline{\boldsymbol{e}}_{3}
$$


We assume that the loading is such that this holds also for the material rotation rate:

$$
\underline{\times} \underline{\underline{w}}=w_{3} \underline{\boldsymbol{e}}_{3}
$$

In this context, the second elasticity law (15) reduces to

$$
\underset{\sim}{\boldsymbol{m}}=2 \beta \underset{\sim}{\boldsymbol{\kappa}}, \quad m_{3 i}=2 \beta \Phi_{3, i}
$$

The internal stress $x$ is then related to the third component of the divergence of the couple stress tensor:

$$
x=-\frac{1}{2}(\operatorname{div} \underset{\sim}{\boldsymbol{m}})_{3}=-\frac{1}{2}\left(m_{31,1}+m_{32,2}\right)=-\beta \Delta \Phi_{3}
$$

where $\Delta$ is the Laplace operator.

The lattice rotation can be expressed in terms of the material and plastic rotation following (21) which becomes:

$$
\underline{\dot{\Phi}}=\underline{\stackrel{x}{\boldsymbol{w}}}^{-\underline{\boldsymbol{w}}^{p}}, \quad \text { with } \quad \underline{\stackrel{\boldsymbol{w}}{w}}^{p}=-\frac{1}{2} \underline{\boldsymbol{\epsilon}}:(\underline{\boldsymbol{l}} \otimes \underline{\boldsymbol{n}})
$$

In the single slip case, the only non-vanishing component is

$$
\dot{\Phi}_{3}=\stackrel{\times}{w}_{3}+\frac{\dot{\gamma}}{2}
$$

Accordingly, the Schmid law (27) can be written as

$$
\tau= \pm \tau_{c}=\tau^{\mathrm{sym}}-x=\tau^{\mathrm{sym}}+\beta \Delta \Phi_{3}
$$

so that

$$
\tau^{\mathrm{sym}}= \pm \tau_{c}-\beta \Delta \Phi_{3}= \pm \tau_{c}-\beta\left(\stackrel{\times}{\omega}_{3}+\frac{1}{2} \Delta \gamma\right)
$$

where $\stackrel{x}{\omega}_{3}$ is obtained by time integration of $\stackrel{x}{w}_{3}$. The internal stress is found to depend on the Laplacian of material rotation and amount of slip.

It is important to check that there is no effect of a pure gradient of slip normal to the slip plane in the model. This is due to the fact that such a gradient of slip does not induce any lattice rotation. To see that, we consider the following kinematics of single glide $\bar{\gamma}\left(x_{2}\right)$ which depends on the coordinate normal to the glide plane $\underline{\boldsymbol{u}}=\bar{\gamma}\left(x_{2}\right) x_{2} \underline{\boldsymbol{e}}_{1}$, where $\bar{\gamma}$ is the prescribed non-homogeneous shear. The gradient of displacement and the plastic deformation take the form

$$
\boldsymbol{\nabla} \underline{\boldsymbol{u}}=\left(\bar{\gamma}_{, 2} x_{2}+\bar{\gamma}\right) \underline{\boldsymbol{e}}_{1} \otimes \underline{\boldsymbol{e}}_{2}, \quad \underline{\boldsymbol{e}}^{p}=\gamma \underline{\boldsymbol{e}}_{1} \otimes \underline{\boldsymbol{e}}_{2}
$$

As a result of equation (36), the lattice rotation is

$$
\Phi_{3}=-\frac{1}{2}\left(\bar{\gamma}_{, 2} x_{2}+\bar{\gamma}\right)+\frac{\gamma}{2}
$$

and the total Cosserat deformation

$$
\begin{aligned}
\underset{\sim}{\boldsymbol{e}} & =\frac{1}{2}\left(\bar{\gamma}, 2 x_{2}+\bar{\gamma}+\gamma\right) \underline{\boldsymbol{e}}_{1} \otimes \underline{\boldsymbol{e}}_{2}-\frac{1}{2}\left(\bar{\gamma}, 2 x_{2}+\bar{\gamma}\right) \underline{\boldsymbol{e}}_{2} \otimes \underline{\boldsymbol{e}}_{1} \\
& =\left(e_{12}^{\boldsymbol{e}}+\gamma\right) \underline{\boldsymbol{e}}_{1} \otimes \underline{\boldsymbol{e}}_{2}+e_{21}^{\boldsymbol{e}} \underline{\boldsymbol{e}}_{2} \otimes \underline{\boldsymbol{e}}_{1}
\end{aligned}
$$




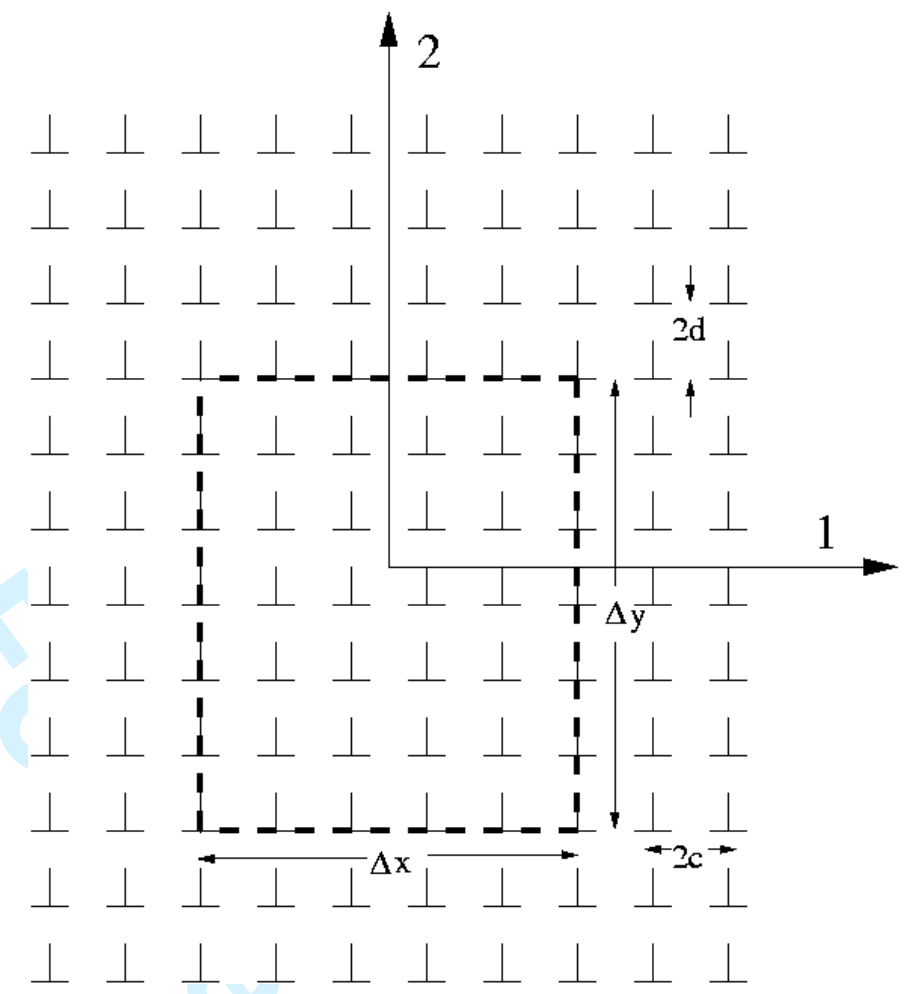

Figure 1. Rectangular array of parallel edge dislocation considered by Kröner [3].

The balance of force stresses requires that the stress component $\sigma_{12}$ does not depend on $x_{2}$, which in turn implies that

$$
e_{12}^{e}=\frac{1}{2}\left(\bar{\gamma}_{, 2} x_{2}+\bar{\gamma}-\gamma\right)=-\Phi_{3}
$$

is constant. The curvature $\kappa_{32}=\Phi_{3,2}$ therefore vanishes.

In other words, this feature of the model shows that the proposed Cosserat theory includes only effect of geometrically necessary dislocations, contrary to the full second gradient model in [5].

\section{Relation to strain gradient plasticity models}

There is a link between lattice curvature and the rotational part of plastic deformation, related to the dislocation density tensor. This relation can be obtained by applying the curl operator to the following expression of total Cosserat deformation rate, within the context of small deformations and rotations:

$$
\underset{\sim}{\dot{e}}=\nabla \underline{\dot{u}}-{\underset{\sim}{w}}^{e}={\underset{\sim}{\dot{e}}}^{e}+{\underset{\sim}{\dot{e}}}^{p}
$$

The elastic deformation ${\underset{\sim}{e}}^{e}$ is symmetric when the constraint (20) that the Cosserat microrotation coincides with lattice rotation is enforced. We get:

$$
-\operatorname{curl} \boldsymbol{\sim}^{e}=\operatorname{curl} \dot{\sim}^{e}+\operatorname{curl}{\stackrel{\sim}{\dot{e}^{p}}}^{\dot{e}}
$$

The curl of the lattice rotation rate is equal to the transpose of the curvature rate tensor by:

$$
\operatorname{curl}{\underset{\sim}{\boldsymbol{w}}}^{e}=\left(\nabla \underline{\boldsymbol{w}}^{e}\right)^{T}=(\nabla \underline{\dot{\Phi}})^{T}={\underset{\sim}{\dot{\boldsymbol{s}}}}^{T}
$$




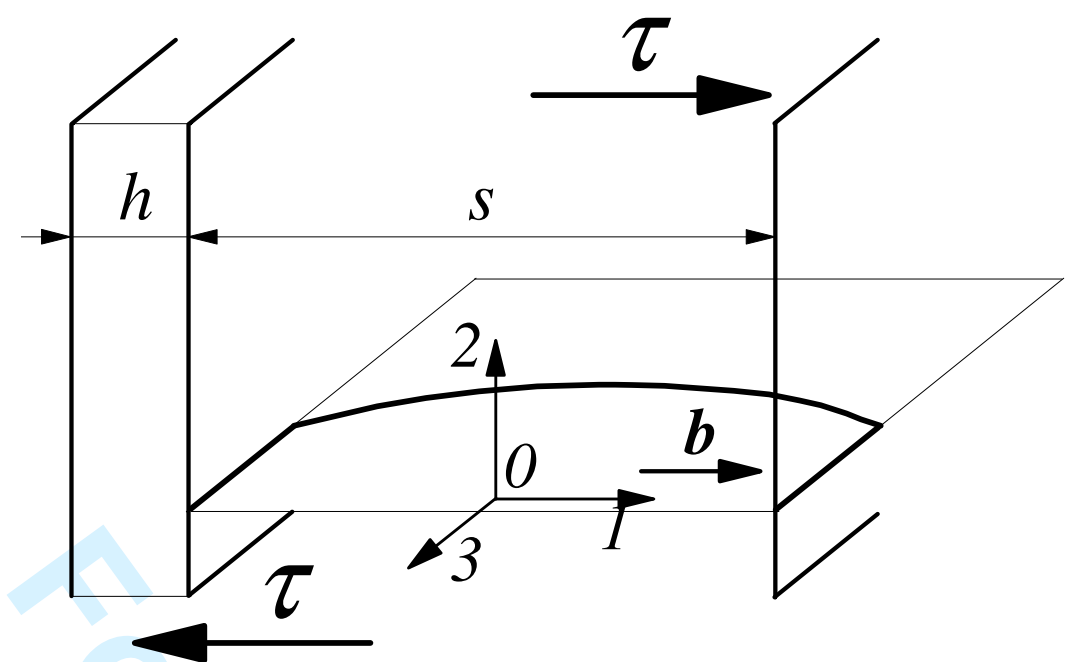

Figure 2. Dislocation bowing in the soft phase. A part of the loop gliding in the plane 1-3 is shown, with the curved (originally screw) section and edge segments at the soft / hard phase interface. The resolved shear stress $\tau$ and Burgers vector $\underline{b}$ are indicated. Labels $s$ and $h$ are used to designate the soft and hard phase, respectively.

In the strain gradient crystal plasticity theories proposed in [7,9], the curl of plastic deformation is introduced into the free energy function. These theories turn out to be identical to the present Cosserat model if the curl of the elastic strain is neglected in (45), as initially done by Nye [6] to derive a direct relation between the dislocation density tensor and lattice curvature.

In the case of single slip, the curl of plastic deformation is computed as:

$$
\left[\operatorname{curl} \dot{\sim}^{p}\right]=\left[\begin{array}{ccc}
0 & 0 & \dot{\gamma}_{, 2} n_{1} n_{2}-\dot{\gamma}_{, 1} n_{2}^{2} \\
0 & 0 & -\dot{\gamma}_{, 2} n_{1}^{2}+\dot{\gamma}_{, 1} n_{1} n_{2} \\
0 & 0 & 0
\end{array}\right]
$$

where $n_{1}$ and $n_{2}, n_{3}=0$ are the components of the normal vector to the slip plane. The couple stress tensor can be approximated by

$$
\underset{\sim}{\boldsymbol{m}}=2 \beta \underset{\sim}{\boldsymbol{\kappa}} \simeq-2 \beta\left(\operatorname{curl}{\underset{\sim}{\boldsymbol{e}}}^{p}\right)^{T}
$$

so that

$$
(\operatorname{div} \underset{\sim}{\boldsymbol{m}})_{3} \simeq-2 \beta\left(2 \gamma_{, 12} n_{1} n_{2}-\gamma_{, 11} n_{2}^{2}-\gamma_{, 22} n_{1}^{2}\right)
$$

and

$$
x=-\frac{1}{2}(\operatorname{div} \underset{\sim}{\boldsymbol{m}})_{3} \simeq \beta\left(2 \gamma_{, 12} n_{1} n_{2}-\gamma_{, 11} n_{2}^{2}-\gamma_{, 22} n_{1}^{2}\right)
$$

This expression, when inserted into (27), gives a yield condition involving the second derivative of the amount of slip in the spirit of the model proposed by [26].

\section{Generalized kinematic hardening modulus}

The material parameter $\beta$ appearing in (34) can be regarded as a generalized kinematic hardening modulus, with the physical dimension MPa.mm². It has been evaluated by Kröner as the bending stiffness of a crystal element containing a rectangular array of edge dislocations. The existence of couple stresses in a dislocated 
crystal was put forward by Kröner [3] by considering the residual stress field after bending and torsion of single crystals. Kröner derived the expression of a material constant linking a couple-stress component to the corresponding lattice curvature tensor component, in the special case depicted in figure 1. This derivation can be interpreted as follows: to maintain a zero plane curvature in a box inside the dislocated crystal of figure 1, a torque must be applied. Conversely, in the absence of couple stresses acting on the volume element, lattice planes are curved due to the excess of dislocations of a definite sign. The characteristic length which goes into this constant is the glide plane distance $d$ between dislocations. Using the present notations, Kröner's relation reads:

$$
m_{31}=\frac{\pi^{2}}{6} \frac{\mu}{1-\nu} d^{2} \kappa_{31}
$$

corresponding to the following value of the parameter $\beta$ from (33):

$$
\beta=\frac{\pi^{2}}{12} \frac{\mu}{1-\nu} d^{2}=\frac{\pi^{2}}{12} \frac{\mu}{1-\nu} \frac{1}{\rho}
$$

where $\mu$ and $\nu$ respectively are the shear modulus and Poisson ratio of the crystal, and $d$ the distance between dislocations estimated as the inverse of the square root of the dislocation density $\rho$ of geometrically necessary dislocations.

The presence of non-homogeneous couple-stresses induces the building up of skew-symmetric force stresses as noticed in [27] following the balance equation (5).

Another interpretation of parameter $\beta$ has been given in the references $[12,13]$ considering a continuous distribution of screw dislocations bowing in a channel undergoing single slip between hard elastic layers, see figure 2. The slip plane is assumed to be perpendicular to the interfaces. During shear in a direction perpendicular to the interfaces, the dislocations continuously bow out in the channel and induce a pile-up of edge parts at the interfaces. It has been shown in $[12,13]$ that the Cosserat model (together with other strain gradient plasticity models) can capture the non-homogeneous distribution of plastic slip in the channel. The Cosserat model has been identified from the dislocation model, with the estimation

$$
\beta=\frac{2 \mu}{\rho}
$$

Let's take for instance: $\mu=40000 \mathrm{MPa}$ and $\rho=10^{12} \mathrm{~m}^{-2}$, we get $\beta=0.08 \mathrm{~Pa} \cdot \mathrm{m}^{2}=0.08 \mathrm{MPa} \cdot \mathrm{mm}^{2}$.

Both dislocation-based models indicate that the characteristic length associated with the overall Cosserat or strain gradient plasticity models:

$$
l_{c}=\sqrt{\frac{\beta}{\mu}}
$$

should not be a constant one but should evolve with deformation. Both models provide an inverse dependence of the generalized kinematic hardening modulus on the dislocation density, that can be used in the phenomenological Cosserat or gradient theories.

\section{Relation to a statistical mechanics based dislocation model}

A statistical theory of dislocations has been developed for single slip in $[28,2]$. It starts from the equation of motion of individual dislocations and shows that the influence of the short range dislocation-dislocation interactions can be well described by a local back-stress that includes a so-called "non-local diffusionlike term", in the spirit of [26]. The model has been compared successfully with two-dimensional discrete dislocation dynamics (DDD) simulations in [14]. A closed set of constitutive equations was formulated and 

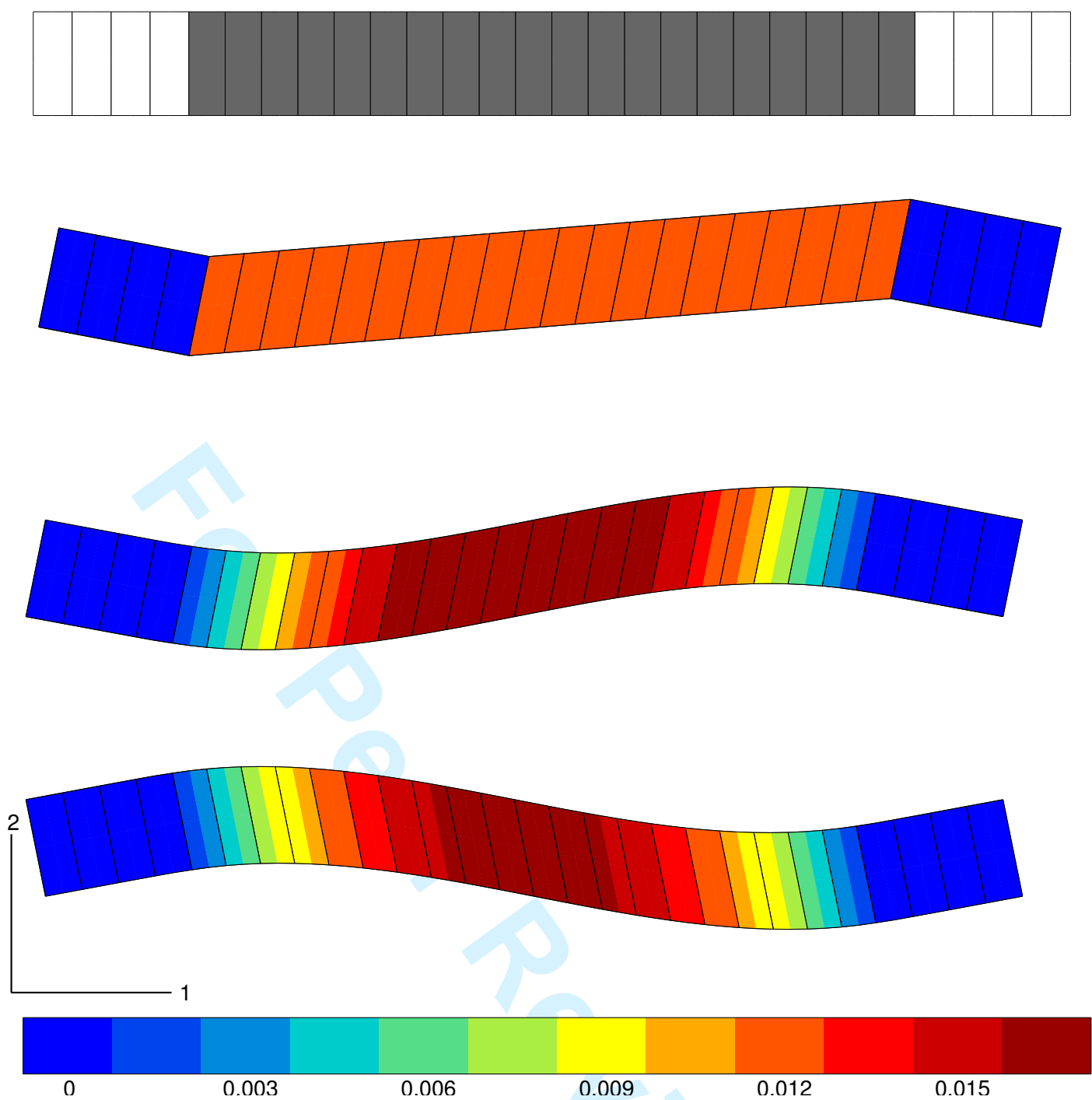

Figure 3. Periodic shearing of a two-phase laminate microstructure. From top to bottom: initial finite element mesh (hard phase in white), prescribed glide $E_{12}=0.01$ according to classical crystal plasticity, prescribed glide $E_{12}=0.01$ according to Cosserat crystal plasticity, prescribed glide $E_{12}=-0.01$ according to Cosserat crystal plasticity. The plotted field is $|\gamma|$. The material parameters are $\mu=26920 \mathrm{MPa}, \tau_{c}=10 \mathrm{MPa}, \beta=10 \mathrm{MPa} \cdot \mathrm{mm}^{2}$.

incorporated into the continuum crystal plasticity framework by these authors in the form:

$$
\begin{aligned}
& \dot{\sim}^{p}=\dot{\gamma} \frac{1}{2}(\underline{\boldsymbol{l}} \otimes \underline{\boldsymbol{n}}+\underline{\boldsymbol{n}} \otimes \underline{\boldsymbol{l}}), \quad \dot{\gamma}=\rho \underline{\boldsymbol{b}} \cdot \underline{\boldsymbol{v}} \\
& \underline{\boldsymbol{v}}=\frac{\underline{\boldsymbol{b}}}{B}(\tau-x) \\
& x=\frac{D \mu \underline{\boldsymbol{b}}}{2 \pi(1-\nu) \rho} \cdot \frac{\partial \kappa}{\partial \underline{\boldsymbol{r}}}
\end{aligned}
$$

where $B, D$ are material parameters. The Burgers vector is $\underline{\boldsymbol{b}}$. The total and excess dislocation densities $\rho=\rho^{+}+\rho^{-}, \kappa=\rho^{+}-\rho^{-}$, fulfill the following evolution partial differential equations:

$$
\dot{\rho}+\operatorname{div}(\kappa \underline{\boldsymbol{v}})=f
$$




$$
\dot{\kappa}+\operatorname{div}(\rho \underline{\boldsymbol{v}})=0
$$

where the function $f$ accounts for dislocation creation and annihilation.

In the case of single slip with $\underline{\boldsymbol{v}}=v \underline{\boldsymbol{e}}_{1}$ and $\underline{\boldsymbol{b}}=b \underline{\boldsymbol{e}}_{1}=b \underline{\boldsymbol{l}}$, the equation (59) reduces to

$$
\dot{\kappa}+\frac{1}{b} \dot{\gamma}_{, 1}=0
$$

so that the back-stress takes the form:

$$
x=-\frac{\alpha \mu}{\rho} \gamma, 11
$$

with $\alpha$ a material parameter. This relation is to be compared to (34) and (50) derived in the Cosserat model. In particular, setting $n_{1}=0$ in (50), i.e. for a slip plane normal to direction 2 , the same dependence on $\gamma_{, 11}$ is retrieved. The inverse dependence of the generalized kinematic hardening modulus with $\rho$ is in accordance with the estimations of the bending stiffness $\beta$ in section 7 , but obtained here in a more general context.

\section{Cyclic plasticity in a two-phase laminate}

The problem of the mechanical behaviour of the two-phase laminate of figure 2 initially studied in the references $[12,13]$, is reexamined here in the context of cyclic deformation and derivation of the resulting global kinematic hardening. Screw dislocations are gliding in a channel along a glide plane perpendicular to the plane parallel interfaces, under the action of a shear stress $\tau=\sigma_{12}$. They deposit edge parts along the interface inducing non-homogeneous plastic deformation in the direction 1 in the channel, and internal stresses associated with the pile-ups. The test is controlled by the prescribed mean total amount of glide $\bar{\gamma}$. The displacement field in the channel is of the form:

$$
\underline{\boldsymbol{u}}=\bar{\gamma} x_{2} \underline{\boldsymbol{e}}_{1}+u_{2}\left(x_{1}\right) \underline{\boldsymbol{e}}_{2}, \quad \underline{\boldsymbol{\Phi}}=\Phi_{3}\left(x_{1}\right) \underline{\boldsymbol{e}}_{3}
$$

with periodicity conditions for the fluctuation $u_{2}\left(x_{1}\right)$ and the micro-rotation $\Phi_{3}$ (see figure 2 for the geometry and coordinate system of the studied microstructure). The hard phase is regarded as an elastic Cosserat medium with a characteristic length much smaller than the one associated with the soft phase. This allows the transmission of the force and couple traction vectors and the continuity of displacement and micro-rotation, at the interface between hard and soft phases. In the elastic hard phase, the microrotation coincides with the material rotation. The active component of the curvature tensor inside the plastic zone is

$$
\kappa_{31}=2 \beta \Phi_{3,1}
$$

According to the balance of momentum equation $\sigma_{21,1}=0$, the stress component $\sigma_{21}$ is constant. The Schmid law (27) indicates that the stress component $\sigma_{12}=\tau_{c}$ is constant. When applied to the equation of balance of moment of momentum (5), and assuming a constant parameter $\beta$, this gives:

$$
m_{31,1}-\sigma_{12}+\sigma_{21}=0 \Longrightarrow m_{31,11}=0 \Longrightarrow \Phi_{3,111}=0
$$

which leads to a parabolic profile of lattice rotation in the channel. This is the same qualitative result as in [13] where however only the symmetric part of the stress was considered for the evaluation of Schmid law. This periodic boundary value problem has also been solved based on the finite element method, as shown in figure 3 for cyclic loading $\bar{\gamma}= \pm 0.01$. In particular the field $\gamma\left(x_{1}\right)$ is given in figure 3 . The curvature effects in the channel are clearly visible. 


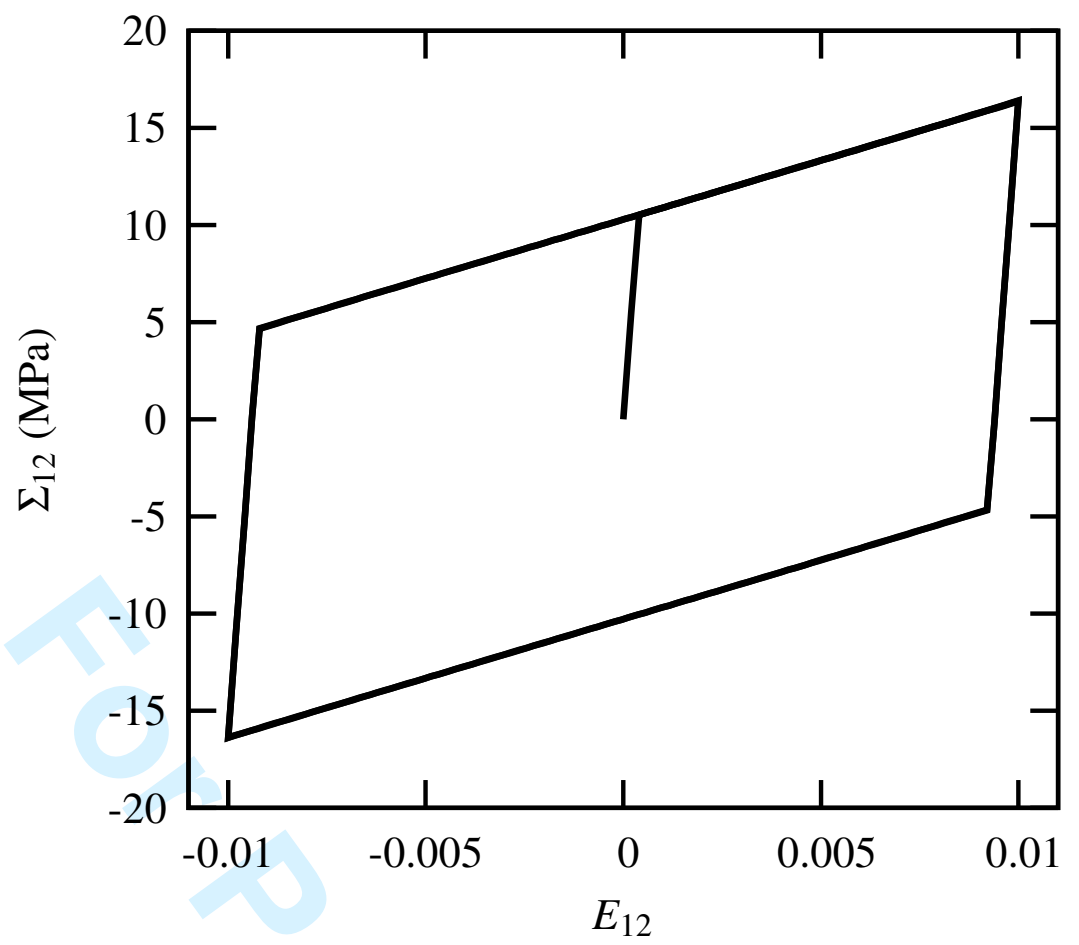

Figure 4. Mean stress-strain curve for the cycling shearing of a two-phase laminate microstructure $\left(\mu=26920 \mathrm{MPa}, \tau_{c}=10 \mathrm{MPa}\right.$, $\left.\beta=10 \mathrm{MPa} \cdot \mathrm{mm}^{2}\right)$.

The effective properties of such a laminate material can be derived from the periodic unit cell by proper averaging procedures. Such homogenization techniques have been developed for heterogeneous Cosserat media in [29]. The nature of the effective medium depends on the ratio of the Cosserat characteristic lengths and on the size of constituents. We use the averaging relations obtained for an effective Cosserat medium:

$$
\Sigma_{12}=<\sigma_{12}>=\frac{1}{V} \int_{V} \sigma_{12} d V, \quad E_{12}=<u_{1,2}>=\frac{1}{V} \int_{V} u_{1,2} d V=\bar{\gamma}
$$

The stress-strain loop obtained for $\bar{\gamma}= \pm 0.01$ is shown in figure 4, revealing effective linear kinematic hardening, induced by the internal stresses in the channel. The observed effective kinematic hardening modulus is found to be directly proportional to the parameter $\beta$.

The generated linear kinematic hardening is similar to the one derived by Tanaka and Mura in the case of a regular distribution of pile-ups in a grain [30], as analyzed in [13]. The kinematic hardening modulus scales with the inverse of the channel width $s$.

\section{Conclusions}

Two main links between available phenomenological generalized continuum crystal plasticity models and the statistical approach to dislocation dynamics have been found:

- In the Cosserat model, the skew-symmetric part of the force stress tensor induces a local back-stress in the generalized Schmid law. This back-stress is a projection of the divergence of couple stresses. A similar contribution exists in strain gradient plasticity models involving the effect of the curl of plastic deformation. In the case of single slip, the latter form of the internal stress is identical to the contribution found in the statistical theory of dislocations of $[28,2]$.

- Dislocation models based either on square arrays of edge dislocations or on the glide of screw dislocations in a channel systematically lead to the existence of a generalized linear kinematic hardening modulus 
which is proportional to the inverse of dislocation density. This dependence can be directly implemented in the phenomenological generalized continuum model. It is also in accordance with the prediction of the statistical approach.

The analysis of the simple case of heterogeneous plastic slip in a channel shows that the internal stresses result in an effective linear classical kinematic hardening on the overall stress-strain curve. Non-linearity can be introduced when the dependence of parameter $\beta$ on dislocation density is implemented. In the given examples, the total dislocation density coincides with the GND content. In more general cases, it is conjectured that the generalized kinematic hardening modulus should depend on the GND part only.

The question arises of the limit case when $\rho$ is going to zero. The kinematic hardening modulus $\beta$ increases for decreasing $\rho$. On the other hand, the curvature is going to zero when density of dislocations in excess decreases. This may result in an indeterminate value of the couple stress according to (33). In the statistical theory of dislocations, it is not excluded that the dislocation density $\rho$ appearing in the expression of back-stress may include parts of both statistical and geometric contents. One may consider the case of a fixed dislocation density $\rho^{S}$ and a vanishing curvature, or equivalently, density $\rho^{G} \rightarrow 0$, which leads to vanishing couple stresses and associated back-stress.

For single slip, most strain gradient plasticity models coincide in predicting a back-stress related to the second derivative of slip in the slip direction. Extensions to the multislip case are currently mainly heuristic and the phenomenological approach based on generalized continua can help formulating them in a consistent manner. Formulations that explicitly introduce the gradient of individual slip or dislocation densities $[15,16,31]$ share the drawback that (dis-) continuity conditions at grain boundaries cannot be formulated unambiguously because the variables $\gamma^{\alpha}, \rho^{\alpha}$ are defined in each grain only up to a crystal symmetry [32]. In contrast, plausible extensions can be proposed based on the rotational part of the full plastic deformation, i.e. the full dislocation density tensor in $[9,17]$, or on the full lattice torsion-curvature tensor $[1,21]$. These extensions have also the advantage that the number of degrees of freedom does not increase when the number of slip systems increases. In the Cosserat model for instance, the number of degrees of freedom remains equal to 3 , the three components of the lattice rotation vector, irrespective of the crystallography of the studied system. The specific crystallographic structure of the studied materials enters the model via the number of internal variables, for instance the number of slip variables $\gamma^{s}$ which depends on the number of slip systems.

\section{References}

[1] S. Forest, G. Cailletaud, and R. Sievert. A Cosserat theory for elastoviscoplastic single crystals at finite deformation. Archives of Mechanics, 49(4):705-736, 1997.

[2] I. Groma, F.F. Csikor, and M. Zaiser. Spatial correlations and higher-order gradient terms in a continuum description of dislocation dynamics. Acta Materialia, 51:1271-1281, 2003.

[3] E. Kröner. On the physical reality of torque stresses in continuum mechanics. Int. J. Engng. Sci., 1:261-278, 1963.

[4] Mura, T. Continuous distribution of dislocations and the mathematical theory of plasticity. Phys. stat. sol., 10:447-453, 1965.

[5] N.A. Fleck and J.W. Hutchinson. Strain gradient plasticity. Adv. Appl. Mech., 33:295-361, 1997.

[6] J.F. Nye. Some geometrical relations in dislocated crystals. Acta Metall., 1:153-162, 1953.

[7] P. Steinmann. Views on multiplicative elastoplasticity and the continuum theory of dislocations. International Journal of Engineering Science, 34:1717-1735, 1996.

[8] S. Forest, R. Sievert, and E.C. Aifantis. Strain gradient crystal plasticity : Thermomechanical formulations and applications. Journal of the Mechanical Behavior of Materials, 13:219-232, 2002.

[9] M.E. Gurtin. A gradient theory of single-crystal viscoplasticity that accounts for geometrically necessary dislocations. Journal of the Mechanics and Physics of Solids, 50:5-32, 2002.

[10] H. Zorski. Statistical theory of dislocations. Int. J. Solids Structures, 4:959-974, 1968.

[11] M. Zaiser, M.C. Miguel, and I. Groma. Statistical dynamics of dislocation systems: The influence of dislocation-dislocation correlations. Phys. Rev. B, 64:224102, 2001.

[12] R. Sedláček and S. Forest. Non-local plasticity at microscale : A dislocation-based model and a Cosserat model. physica status solidi (b), 221:583-596, 2000.

[13] S. Forest and R. Sedláček. Plastic slip distribution in two-phase laminate microstructures: Dislocation-based vs. generalizedcontinuum approaches. Philosophical Magazine A, 83:245-276, 2003.

[14] S. Yefimov, I. Groma, and E. Van der Giessen. A comparison of a statistical-mechanics based plasticity model with discrete dislocation plasticity calculations. J. Mech. Phys. Solids, 52:279-300, 2004.

[15] S. Yefimov and E. Van der Giessen. Multiple slip in a strain-gradient plasticity model motivated by a statistical-mechanics description of dislocations. International Journal of Solids and Structures, 42:3375-3394, 2005.

[16] C.J. Bayley, W.A.M. Brekelmans, and M.G.D. Geers. A comparison of dislocation induced back stress formulations in strain gradient crystal plasticity. International Journal of Solids and Structures, 43:7268-7286, 2006.

[17] E.M. Viatkina, W.A.M. Brekelmans, and M.G.D. Geers. Modelling of the internal stress in dislocation cell structures. European Journal of Mechanics A/solids, 26:982-998, 2007. 
[18] P. Cermelli and M.E. Gurtin. On the characterization of geometrically necessary dislocations in finite plasticity. Journal of the Mechanics and Physics of Solids, 49:1539-1568, 2001.

[19] B. Svendsen. Continuum thermodynamic models for crystal plasticity including the effects of geometrically-necessary dislocations. J. Mech. Phys. Solids, 50:1297-1329, 2002.

[20] S. Forest and R. Sievert. Elastoviscoplastic constitutive frameworks for generalized continua. Acta Mechanica, 160:71-111, 2003.

[21] S. Forest, F. Barbe, and G. Cailletaud. Cosserat modelling of size effects in the mechanical behaviour of polycrystals and multiphase materials. International Journal of Solids and Structures, 37:7105-7126, 2000.

[22] R. de Borst. Simulation of strain localization: a reappraisal of the Cosserat continuum. Engng Computations, 8:317-332, 1991.

[23] C. Teodosiu. Large plastic deformation of crystalline aggregates. CISM Courses and Lectures No. 376, Udine, Springer Verlag, Berlin, 1997.

[24] W.T. Koiter. Couple-stresses in the theory of elasticity. i and ii. Proc. K. Ned. Akad. Wet., B67:17-44, 1963.

[25] H. Gao, Y. Huang, W.D. Nix, and J.W. Hutchinson. Mechanism-based strain gradient plasticity - i. theory. J. Mech. Phys. Solids, 47:1239-1263, 1999

[26] E.C. Aifantis. The physics of plastic deformation. International Journal of Plasticity, 3:211-248, 1987.

[27] F. Hehl and E. Kröner. Zum Materialgesetz eines elastischen Mediums mit Momentenspannungen. Z. Naturforschg., 20a:336-350, 1958.

[28] I. Groma. Link between the microscopic and mesoscopic length-scale description of the collective behaviour of dislocations. Phys. Rev. B, 56:5807-5813, 1997.

[29] S. Forest, F. Pradel, and K. Sab. Asymptotic analysis of heterogeneous Cosserat media. International Journal of Solids and Structures, 38:4585-4608, 2001.

[30] K. Tanaka and T. Mura. A dislocation model for fatigue crack initiation. Journal of Applied Mechanics, 48:97-103, 1981.

[31] C.J. Bayley, W.A.M. Brekelmans, and M.G.D. Geers. A three-dimensional dislocation field crystal plasticity approach applied to miniaturized structures. Philosophical Magazine, 87:1361-1378, 2007.

[32] S. Forest. The micromorphic approach for gradient elasticity, viscoplasticity and damage. ASCE Journal of Engineering Mechanics, in press, 2008. 


\title{
Some links between Cosserat, strain gradient crystal plasticity and the statistical theory of dislocations
}

\author{
Samuel FOREST* \\ Mines Paris, ParisTech, CNRS UMR 7633 \\ BP 87, 91003 Evry Cedex, France \\ (revised)
}

\begin{abstract}
A link is established between a phenomenological Cosserat model of crystal plasticity due to [1] and recent results obtained in the statistical theory of dislocations by [2]. The existence of a back-stress related to the divergence of the couple stress tensor is derived. According to several dislocation-based models of single slip, the kinematic hardening modulus is found to be inversely proportional to dislocation density. Phenomenological extensions to multislip situations can be proposed based on these generalized continuum approaches.
\end{abstract}

\section{Introduction}

First links between the continuum theory of dislocations and the mechanics of generalized continua were established in the pioneering works of Kröner and Mura [3,4]. The connection between the dislocation density tensor and the rotational part of the plastic deformation was an incentive for the development of strain gradient crystal plasticity theories, based on the second gradient medium as proposed in [5]. On the other hand, the relation between the dislocation density tensor and lattice curvature derived by Nye [6] prompted several authors as recorded in [1] to formulate a Cosserat model for crystal plasticity. Since then a plethora of strain gradient plasticity models have been proposed without clear classification nor guidelines for assessing the fundamental differences between them. One of the objectives of this work is to show that the Cosserat theory and strain gradient plasticity models based on the introduction of the curl of plastic strain share major common features and belong to the same class of generalized crystal plasticity models.

Recent formulations of generalized continuum models incorporating the effect of the curl of plastic deformation within the framework of continuum thermomechanics show that this additional contribution to hardening compared to classical crystal plasticity, arises as a gradient-dependent internal stress or back-stress in the constitutive equations $[7,8,9]$.

In the mean time, the statistical theory of dislocations has made significant progress since the early attempts like [10]. In particular, higher order gradient terms in the continuum description have been derived in $[11,2]$ from spatial correlations of short-range dislocation-dislocation interactions, at least in the case of populations of straight parallel edge dislocations. This contribution was shown to be adequately represented by a back-stress component which is inversely proportional to the dislocation density. Closely related scaling laws were established for specific distributions of dislocations in relation to the Cosserat continuum theory $[3,12,13]$.

Comparisons with numerical simulations based on two-dimensional dislocation dynamics support the pertinence of this formulation for gradient-dependent internal stresses [14]. This contribution is essentially proportional to the second derivative of slip in the slip direction. The objective of the present work is to show that the mentioned Cosserat and strain gradient plasticity models lead to the same formulation of internal stresses, from purely phenomenological arguments, thus bridging a gap between statistical and constitutive theories, at least in the case of single slip.

*Corresponding author. Email: samuel.forest@ensmp.fr

Philosophical Magazine

ISSN 1478-6435 print/ISSN 1478-6443 online (c) 200x Taylor \& Francis

http://www.tandf.co.uk/journals

DOI: $\operatorname{xxxxxxxx}$ 
Extensions of the continuum model to multiple slip currently remain on a heuristic basis. Such extensions were recently proposed in $[15,16]$. The phenomenological constitutive approach making use of generalized continua also systematically provides such 3D formulations. It will be shown in the present work that the phenomenological and statistical approaches share several common features in the case of single slip. Accordingly, it is worth comparing the 3D formulations of both classes of models. Such gradient dependent internal stresses can be embedded in more comprehensive and realistic constitutive models for the deformation of metals and alloys, as shown recently in [17].

We start to recall the Cosserat crystal plasticity framework focusing on the relation between the Cosserat microrotation and crystal lattice rotation. In particular, one additional elastic parameter of the Cosserat model will be interpreted as a Lagrange multiplier to make both rotations coincide. A Schmid law is then formulated in section 4 based on the generally non-symmetric Cosserat force stress tensor. It is shown to lead to the existence of a back-stress expressed in terms of couple stresses. The found internal stresses are explicited in the case of single slip in section 5 and compared to results from statistical description of dislocation behaviour in section 8 .

The derivations of the generalized continuum models are presented within the small deformation framework for the sake of simplicity. Extensions to finite deformation formulations are straightforward following the lines of $[1,18,19,20]$.

In this work, zeroth, first, second, third and fourth order tensors are denoted by $a, \underset{\boldsymbol{a}}{\boldsymbol{a}} \underset{\sim}{\boldsymbol{a}} \underset{\sim}{\boldsymbol{a}}, \underset{\sim}{\boldsymbol{a}}$ respectively. The simple and double contractions are written ., .. In index form with respect to an orthonormal Cartesian basis, these notations correspond to $\underline{\boldsymbol{a}} \cdot \underline{\boldsymbol{b}}=a_{i} b_{i}, \quad \underset{\sim}{\boldsymbol{a}}: \underset{\sim}{\boldsymbol{b}}=a_{i j} b_{i j}$, where repeated indices are summed up. The tensor product is denoted by $\otimes$. The nabla operator is denoted by $\boldsymbol{\nabla}$. For example, the component $i j k$ of $\boldsymbol{\nabla} \underset{\sim}{\boldsymbol{A}}$ is $A_{i j, k}$. For full clarity, both intrinsic and index notations are given at several places in the text.

\section{Cosserat crystal plasticity at small deformation}

The degrees of freedom of the theory are the displacement vector $\underline{\boldsymbol{u}}$ and the rotation pseudo-vector $\underline{\boldsymbol{\Phi}}$ which is associated with the skew-symmetric part of the micro-rotation tensor $\underset{\sim}{\boldsymbol{R}}$ in the case of small rotations:

$$
\underset{\sim}{\boldsymbol{R}} \simeq \underset{\sim}{\mathbf{1}}-\underset{\sim}{\boldsymbol{\epsilon}} \cdot \underline{\boldsymbol{\Phi}}, \quad R_{i j} \simeq \delta_{i j}-\epsilon_{i j k} \Phi_{k}
$$

where $\epsilon_{i j k}$ denotes the permutation tensor. The deformation measures of the Cosserat theory are the relative deformation tensor $\underset{\sim}{e}$ and the torsion-curvature tensor $\underset{\sim}{\kappa}$ :

$$
\begin{gathered}
\underset{\sim}{e}=\boldsymbol{\nabla} \underline{\boldsymbol{u}}+\underset{\sim}{\boldsymbol{\epsilon}} \cdot \underline{\Phi}, \quad \underset{\sim}{\boldsymbol{\kappa}}=\nabla \underline{\boldsymbol{\Phi}} \\
e_{i j}=u_{i, j}+\epsilon_{i j k} \Phi_{k}, \quad \kappa_{i j}=\Phi_{i, j}
\end{gathered}
$$

The stress measures associated with the deformation rates in the power of internal forces are the force stress tensor $\boldsymbol{\sigma}$ and the couple stress tensor $\underset{\sim}{\boldsymbol{m}}$. They are generally not symmetric. They must satisfy the balance equations for momentum and balance of momentum, written in the static case:

$$
\operatorname{div} \underset{\sim}{\boldsymbol{\sigma}}=0, \quad \sigma_{i j, j}=0
$$

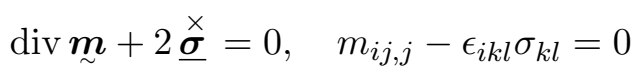


where volume forces and couples are excluded for brevity. The axial vector associated with the skewsymmetric part of the stress tensor is denoted by:

$$
\stackrel{\times}{\underline{\boldsymbol{\sigma}}}=-\frac{1}{2} \underset{\underline{\epsilon}}{\boldsymbol{\epsilon}}: \underset{\sim}{\boldsymbol{\sigma}}, \quad \stackrel{\times}{\sigma}_{i}=-\frac{1}{2} \epsilon_{i k l} \sigma_{k l}
$$

It couples the balance of momentum and moment of momentum equations. Exponents ${ }^{s}$ and ${ }^{a}$ are introduced to respectively denote the symmetric and skew-symmetric parts of the corresponding tensor:

$$
\begin{gathered}
\underset{\sim}{\boldsymbol{\sigma}}={\underset{\sim}{\boldsymbol{\sigma}}}^{s}+{\underset{\sim}{\boldsymbol{\sigma}}}^{a}, \quad \stackrel{\boldsymbol{\sigma}}{a}^{a}=-\underset{\sim}{\boldsymbol{\epsilon}} \cdot \stackrel{\stackrel{\times}{\boldsymbol{\sigma}}}{=} \frac{1}{2} \underset{\sim}{\boldsymbol{\epsilon}} \cdot \underset{\underline{\epsilon}}{\boldsymbol{\epsilon}}: \underset{\sim}{\boldsymbol{\sigma}} \\
\sigma_{i j}^{a}=-\frac{1}{2} \epsilon_{i j k} \stackrel{\times}{\sigma}_{k}=\frac{1}{2} \epsilon_{i j k} \epsilon_{k m n} \sigma_{m n}
\end{gathered}
$$

The traction vector $\underline{\boldsymbol{t}}$ and couple stress vector $\underline{\boldsymbol{m}}$ acting on a surface element must fulfill the following boundary conditions:

$$
\begin{gathered}
\underline{\boldsymbol{t}}=\underset{\sim}{\boldsymbol{\sigma}} \cdot \underline{\boldsymbol{n}}, \quad t_{i}=\sigma_{i j} n_{j} \\
\underline{\boldsymbol{m}}=\underset{\sim}{\boldsymbol{m}} \underline{\underline{\boldsymbol{n}}}, \quad m_{i}=m_{i j} n_{j}
\end{gathered}
$$

where $\underline{\boldsymbol{n}}$ is the outward oriented normal vector at any point of the boundary of the body. The relative deformation can be split into elastic and plastic parts,

$$
\underset{\sim}{e}={\underset{\sim}{e}}^{e}+\stackrel{\sim}{e}^{p}, \quad e_{i j}=e_{i j}^{e}+e_{i j}^{p}
$$

Such a decomposition is not introduced in this work for the total curvature $\underset{\sim}{\boldsymbol{\sim}}$ for the sake of simplicity. A partition of curvature was considered in $[1,21]$ and it is not recalled here. The elastic deformation and the total curvature tensors are assumed to be linked with the force and couple stress tensors, respectively, by a generalized Hooke law:

$$
\begin{aligned}
& \underset{\sim}{\boldsymbol{\sigma}}=\underset{\sim}{\boldsymbol{E}}:{\underset{\sim}{e}}^{e}, \quad \sigma_{i j}=E_{i j k l} e_{k l}^{e} \\
& \underset{\sim}{\boldsymbol{m}}=\underset{\sim}{\boldsymbol{C}}: \underset{\sim}{\boldsymbol{\kappa}}, \quad m_{i j}=C_{i j k l} \kappa_{k l}
\end{aligned}
$$

where $\underset{\sim}{\boldsymbol{E}}$ is the fourth order tensor of elastic moduli (unit $\mathrm{MPa}$ ) and $\underset{\approx}{\boldsymbol{C}}$ are secant moduli of torsion and bending stiffness (unit MPa.mm²). In the isotropic case, these tensors are built from 6 independent elastic moduli:

$$
\begin{gathered}
\underset{\sim}{\boldsymbol{\sigma}}=\lambda\left(\operatorname{trace}{\underset{\sim}{\boldsymbol{e}}}^{e}\right) \underset{\sim}{\mathbf{1}}+2 \mu{\underset{\sim}{\boldsymbol{e}}}^{e s}+2 \mu_{c} \stackrel{\sim}{\boldsymbol{e}}^{e a} \\
\underset{\sim}{\boldsymbol{m}}=\alpha(\operatorname{trace} \underset{\sim}{\boldsymbol{\kappa}}) \underset{\sim}{\mathbf{1}}+2 \beta{\underset{\sim}{\boldsymbol{\kappa}}}^{s}+2 \gamma \underset{\sim}{\boldsymbol{\kappa}}
\end{gathered}
$$

The Lamé constants are $\lambda$ and $\mu$. The coupling modulus $\mu_{c}$ relates the skew-symmetric part of the relative deformation tensor to the skew-symmetric part of the stress tensor. The additional Cosserat parameters $\alpha, \beta, \gamma$ are intrinsic torsion and bending stiffnesses. We adopt the simplification $\beta=\gamma$ as in [22]. 
The kinematics of plastic flow is dictated by the orientation tensors $\underset{\sim}{\boldsymbol{P}}{ }^{s}$ associated with the crystallography of the $N$ slip systems:

$$
\stackrel{\dot{e}}{p}^{p}=\sum_{\alpha=1}^{N} \dot{\gamma}^{\alpha}{\underset{\sim}{\boldsymbol{P}}}^{\alpha}, \quad \text { with } \quad{\underset{\sim}{\boldsymbol{P}}}^{\alpha}=\underline{\boldsymbol{l}}^{\alpha} \otimes \underline{\boldsymbol{n}}^{\alpha}
$$

where $\underline{\boldsymbol{l}}^{\alpha}$ and $\underline{\boldsymbol{n}}^{\alpha}$ respectively are the slip direction and the normal to the slip plane vectors for slip system number $\alpha$. For each slip system, the increment of plastic slip is $\dot{\gamma}^{\alpha}$.

\section{Cosserat microrotation and lattice rotation}

The lattice rotation rate $\boldsymbol{w}^{e}$ is defined as the difference between the material rotation rate $\underset{\sim}{\boldsymbol{w}}$ and the plastic rotation rate represented by the skew-symmetric part of plastic deformation [23]:

$$
\begin{aligned}
& \underline{\sim}^{e}=(\boldsymbol{\nabla} \underline{\dot{u}})^{a}-\underline{\sim}^{p a} \\
& =\stackrel{\sim}{\dot{e}}^{a}-\underset{\sim}{\boldsymbol{\epsilon}} . \underline{\dot{\Phi}}-\stackrel{\dot{e}}{p a}^{p a} \\
& =\stackrel{\dot{e}}{e}^{e a}-\underset{\sim}{\epsilon} \cdot \underline{\dot{\Phi}}
\end{aligned}
$$

which provides a relation between the lattice rotation rate ${\underset{\sim}{\boldsymbol{w}}}^{e}$ and the Cosserat microrotation rate $-\underset{\sim}{\boldsymbol{\epsilon}} \underline{\dot{\Phi}}$. Accordingly, the Cosserat microrotation can be identified with lattice rotation if and only if the following internal constraint is enforced:

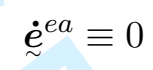

that is when the skew-symmetric part of the elastic relative deformation vanishes. In the works [21, 13], this constraint was enforced by a penalty method which consists in setting a high enough value of the constitutive parameter $\mu_{c}$. This parameter could also be treated as a Lagrange multiplier to ensure the identification between Cosserat rotation and lattice rotation. In that case, the skew-symmetric part of the force stress tensor must be regarded as a reaction force associated with the internal constraint. A similar (but different) situation is met in the so-called Koiter or couple stress theory for which the Cosserat microrotation coincides with the material rotation itself [24].

When the constraint (20) is enforced, the Cosserat directors are lattice vectors. In that case, the following relationship is obtained between Cosserat, material and lattice rotations:

$$
-\underline{\boldsymbol{\epsilon}} \cdot \underline{\dot{\Phi}}=\boldsymbol{w}^{e}=\underset{\sim}{\boldsymbol{w}}-\boldsymbol{w}^{p}
$$

where $\underset{\sim}{\boldsymbol{w}}=(\boldsymbol{\nabla} \underline{\dot{\boldsymbol{u}}})^{a}, \boldsymbol{\sim}^{p}={\underset{\sim}{\dot{e}}}^{p a}$ are the skew-symmetric parts of the total and plastic deformation rates respectively. The associated rotation vectors therefore satisfy the relation:

$$
\underline{\dot{\Phi}}=\underline{x}^{\stackrel{\times}{\boldsymbol{w}}} \underline{\underline{w}}^{p}
$$

\section{Schmid law with a non-symmetric stress tensor}

Plastic slip is activated when the resolved shear stress on a given slip system reaches a critical value. The resolved shear stress $\tau^{s}$ is the component of the traction vector acting on the slip plane in the slip direction:

$$
\begin{aligned}
\tau^{\alpha} & =\left(\underset{\sim}{\boldsymbol{\sigma}} \cdot \underline{\boldsymbol{n}}^{\alpha}\right) \cdot \underline{\boldsymbol{l}}^{\alpha}=\underset{\sim}{\boldsymbol{\sigma}}:{\underset{\sim}{\boldsymbol{P}}}^{\alpha}=\boldsymbol{\sigma}^{s}:{\underset{\sim}{\boldsymbol{P}}}^{\alpha s}+{\underset{\sim}{\boldsymbol{\sigma}}}^{a}:{\underset{\sim}{\boldsymbol{P}}}^{\alpha a} \\
& =\tau^{\mathrm{sym} \alpha}-x^{\alpha}
\end{aligned}
$$


A decomposition of the total resolved shear stress is found into a component $\tau^{\text {sym }}$ which is nothing but the resolved shear stress computed with the symmetrized stress tensor, and an internal stress variable $x^{s}$ defined as:

$$
x^{\alpha}=-\underline{\sim}^{a}: \underline{\sim}^{\alpha a}=\underline{\boldsymbol{\sigma}}^{\times} \cdot\left(\underline{\boldsymbol{l}}^{\alpha} \wedge \underline{\boldsymbol{n}}^{\alpha}\right)
$$

where $\wedge$ denotes the vector product. The additional contribution associated with the skew-symmetric part of the stress will therefore act as a back-stress in the yield criterion. An alternative expression of the internal stress can be worked out by taking the balance of moment of momentum equation (5) into account:

$$
x^{\alpha}=-\frac{1}{2}(\operatorname{div} \underset{\sim}{\boldsymbol{m}}) \cdot\left(\underline{\boldsymbol{l}}^{\alpha} \wedge \underline{\boldsymbol{n}}^{\alpha}\right)
$$

which relates the internal stress $x^{\alpha}$ to a projection of the divergence of the couple stress tensor.

The slip activation criterion for slip system $\alpha$ is

$$
f^{\alpha}\left(\underset{\sim}{\boldsymbol{\sigma}}, \tau_{c}^{\alpha}\right)=\left|\tau^{\alpha}\right|-\tau_{c}^{\alpha}=\left|\tau^{\mathrm{sym} \alpha}-x^{s}\right|-\tau_{c}^{\alpha}=0
$$

This criterion involves the critical resolved shear stress $\tau_{c}^{\alpha}$ for which usual hardening laws of crystal plasticity can be used. At this stage, the evolution of $\tau_{c}^{\alpha}$ can be limited to the effect of so-called statistically stored dislocations (density $\rho^{S}$ ). The effect of the dislocation density tensor, or equivalently so-called geometrically necessary dislocation densities (GND), enters the model via the back-stress $x^{s}$ related to couple stresses and therefore to lattice curvature, as a result of the celebrated Nye relation connecting the dislocation density tensor and lattice curvature [6]. This is at variance with the quite common modification of Taylor's rule, namely $\tau_{c}$ proportional to $\sqrt{\rho^{S}+\rho^{G}}$, where different dislocation density measures are combined in the friction stress [25].

A viscoplastic flow rule is adopted to compute the amount of plastic glide:

$$
\dot{\gamma}=\left\langle\frac{f}{K}\right\rangle^{n} \operatorname{sign}\left(\tau^{\alpha}\right)
$$

in the form of a power law involving the viscosity parameter $K$ and the power $n$. The brackets denote the positive part of the quantity.

\section{$5 \quad$ Application to single slip}

We derive here the special form of the previous constitutive and balance equations when plastic deformation proceeds through single slip in a plane of normal $\underline{\boldsymbol{n}}$ in the direction $\underline{\boldsymbol{l}}$ :

$$
\stackrel{\sim}{\underline{e}}^{p}=\dot{\gamma} \underline{\boldsymbol{l}} \otimes \underline{\boldsymbol{n}}
$$

We adopt a Cartesian coordinate system such that the third vector of the basis is

$$
\underline{\boldsymbol{e}}_{3}=\underline{\boldsymbol{l}} \wedge \underline{\boldsymbol{n}}
$$

We assume that no gradient will develop along the out of plane direction. In this two-dimensional situation, the lattice rotation will take place with respect to the third axis:

$$
\underline{\Phi}=\Phi_{3} \underline{\boldsymbol{e}}_{3}
$$


We assume that the loading is such that this holds also for the material rotation rate:

$$
\underline{\times} \underline{\underline{w}}=w_{3} \underline{\boldsymbol{e}}_{3}
$$

In this context, the second elasticity law (15) reduces to

$$
\underset{\sim}{\boldsymbol{m}}=2 \beta \underset{\sim}{\boldsymbol{\kappa}}, \quad m_{3 i}=2 \beta \Phi_{3, i}
$$

The internal stress $x$ is then related to the third component of the divergence of the couple stress tensor:

$$
x=-\frac{1}{2}(\operatorname{div} \underset{\sim}{\boldsymbol{m}})_{3}=-\frac{1}{2}\left(m_{31,1}+m_{32,2}\right)=-\beta \Delta \Phi_{3}
$$

where $\Delta$ is the Laplace operator.

The lattice rotation can be expressed in terms of the material and plastic rotation following (21) which becomes:

$$
\underline{\dot{\Phi}}=\underline{\stackrel{x}{\boldsymbol{w}}}^{-\underline{\boldsymbol{w}}^{p}}, \quad \text { with } \quad \underline{\stackrel{\boldsymbol{w}}{w}}^{p}=-\frac{1}{2} \underline{\boldsymbol{\epsilon}}:(\underline{\boldsymbol{l}} \otimes \underline{\boldsymbol{n}})
$$

In the single slip case, the only non-vanishing component is

$$
\dot{\Phi}_{3}=\stackrel{\times}{w}_{3}+\frac{\dot{\gamma}}{2}
$$

Accordingly, the Schmid law (27) can be written as

$$
\tau= \pm \tau_{c}=\tau^{\mathrm{sym}}-x=\tau^{\mathrm{sym}}+\beta \Delta \Phi_{3}
$$

so that

$$
\tau^{\mathrm{sym}}= \pm \tau_{c}-\beta \Delta \Phi_{3}= \pm \tau_{c}-\beta\left(\stackrel{\times}{\omega}_{3}+\frac{1}{2} \Delta \gamma\right)
$$

where $\stackrel{x}{\omega}_{3}$ is obtained by time integration of $\stackrel{x}{w}_{3}$. The internal stress is found to depend on the Laplacian of material rotation and amount of slip.

It is important to check that there is no effect of a pure gradient of slip normal to the slip plane in the model. This is due to the fact that such a gradient of slip does not induce any lattice rotation. To see that, we consider the following kinematics of single glide $\bar{\gamma}\left(x_{2}\right)$ which depends on the coordinate normal to the glide plane $\underline{\boldsymbol{u}}=\bar{\gamma}\left(x_{2}\right) x_{2} \underline{\boldsymbol{e}}_{1}$, where $\bar{\gamma}$ is the prescribed non-homogeneous shear. The gradient of displacement and the plastic deformation take the form

$$
\boldsymbol{\nabla} \underline{\boldsymbol{u}}=\left(\bar{\gamma}_{, 2} x_{2}+\bar{\gamma}\right) \underline{\boldsymbol{e}}_{1} \otimes \underline{\boldsymbol{e}}_{2}, \quad \underline{\boldsymbol{e}}^{p}=\gamma \underline{\boldsymbol{e}}_{1} \otimes \underline{\boldsymbol{e}}_{2}
$$

As a result of equation (36), the lattice rotation is

$$
\Phi_{3}=-\frac{1}{2}\left(\bar{\gamma}_{, 2} x_{2}+\bar{\gamma}\right)+\frac{\gamma}{2}
$$

and the total Cosserat deformation

$$
\begin{aligned}
\underset{\sim}{\boldsymbol{e}} & =\frac{1}{2}\left(\bar{\gamma}, 2 x_{2}+\bar{\gamma}+\gamma\right) \underline{\boldsymbol{e}}_{1} \otimes \underline{\boldsymbol{e}}_{2}-\frac{1}{2}\left(\bar{\gamma}, 2 x_{2}+\bar{\gamma}\right) \underline{\boldsymbol{e}}_{2} \otimes \underline{\boldsymbol{e}}_{1} \\
& =\left(e_{12}^{\boldsymbol{e}}+\gamma\right) \underline{\boldsymbol{e}}_{1} \otimes \underline{\boldsymbol{e}}_{2}+e_{21}^{\boldsymbol{e}} \underline{\boldsymbol{e}}_{2} \otimes \underline{\boldsymbol{e}}_{1}
\end{aligned}
$$




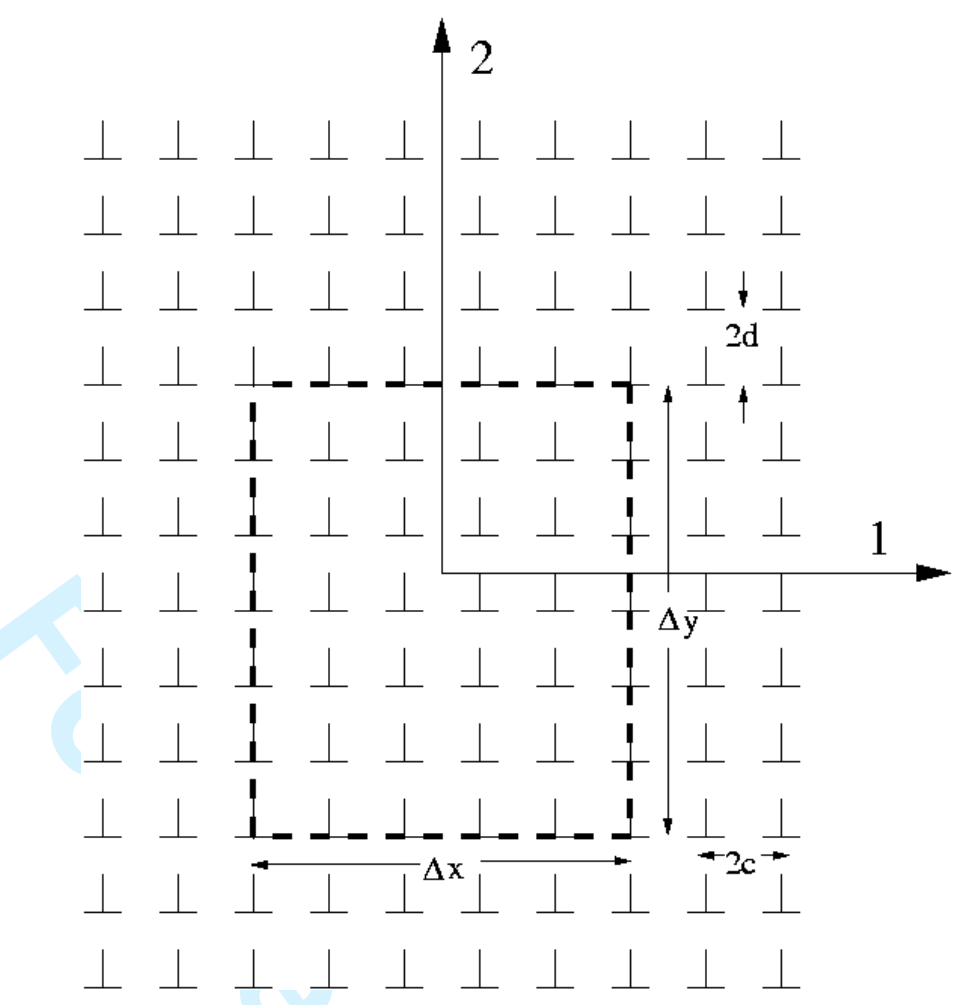

Figure 1. Rectangular array of parallel edge dislocation considered by Kröner [3].

The balance of force stresses requires that the stress component $\sigma_{12}$ does not depend on $x_{2}$, which in turn implies that

$$
e_{12}^{e}=\frac{1}{2}\left(\bar{\gamma}_{, 2} x_{2}+\bar{\gamma}-\gamma\right)=-\Phi_{3}
$$

is constant. The curvature $\kappa_{32}=\Phi_{3,2}$ therefore vanishes.

In other words, this feature of the model shows that the proposed Cosserat theory includes only effect of geometrically necessary dislocations, contrary to the full second gradient model in [5].

\section{$6 \quad$ Relation to strain gradient plasticity models}

There is a link between lattice curvature and the rotational part of plastic deformation, related to the dislocation density tensor. This relation can be obtained by applying the curl operator to the following expression of total Cosserat deformation rate, within the context of small deformations and rotations:

$$
\underset{\sim}{\dot{e}}=\nabla \underline{\dot{u}}-{\underset{\sim}{w}}^{e}={\underset{\sim}{\dot{e}}}^{e}+{\underset{\sim}{\dot{e}}}^{p}
$$

The elastic deformation ${\underset{\sim}{e}}^{e}$ is symmetric when the constraint (20) that the Cosserat microrotation coincides with lattice rotation is enforced. We get:

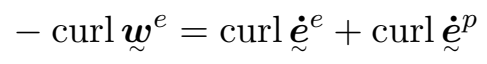

The curl of the lattice rotation rate is equal to the transpose of the curvature rate tensor by:

$$
\operatorname{curl}{\underset{\sim}{\boldsymbol{w}}}^{e}=\left(\boldsymbol{\nabla} \underline{\boldsymbol{w}}^{e}\right)^{T}=(\nabla \underline{\dot{\Phi}})^{T}=\dot{\sim}^{T}
$$




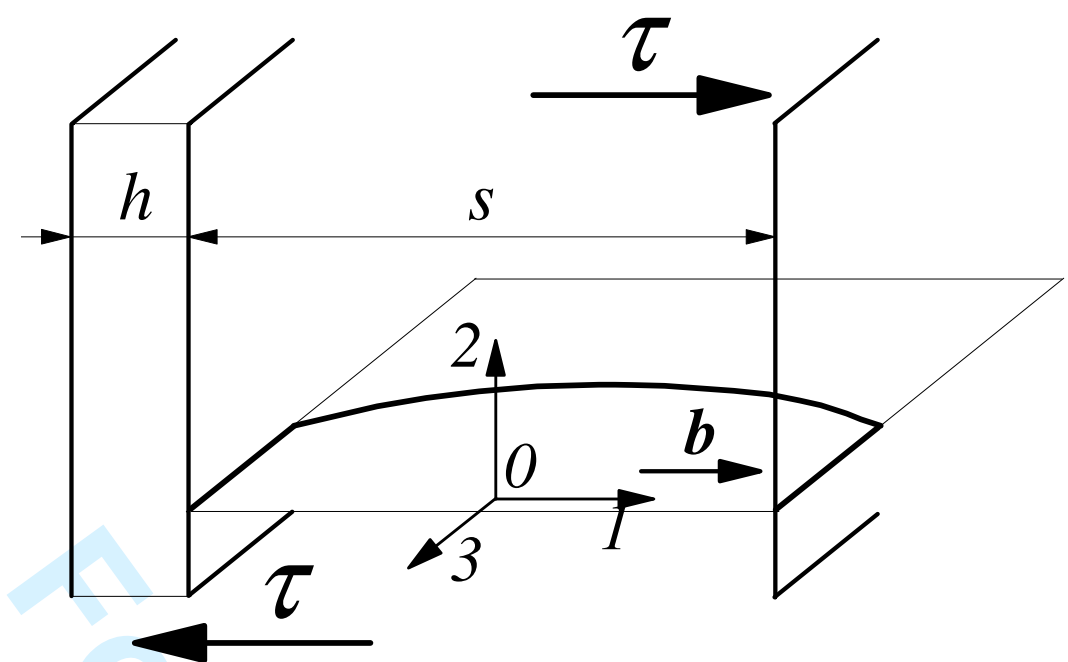

Figure 2. Dislocation bowing in the soft phase. A part of the loop gliding in the plane 1-3 is shown, with the curved (originally screw) section and edge segments at the soft / hard phase interface. The resolved shear stress $\tau$ and Burgers vector $\underline{b}$ are indicated. Labels $s$ and $h$ are used to designate the soft and hard phase, respectively.

In the strain gradient crystal plasticity theories proposed in [7,9], the curl of plastic deformation is introduced into the free energy function. These theories turn out to be identical to the present Cosserat model if the curl of the elastic strain is neglected in (45), as initially done by Nye [6] to derive a direct relation between the dislocation density tensor and lattice curvature.

In the case of single slip, the curl of plastic deformation is computed as:

$$
\left[\operatorname{curl} \dot{\sim}^{p}\right]=\left[\begin{array}{ccc}
0 & 0 & \dot{\gamma}_{, 2} n_{1} n_{2}-\dot{\gamma}_{, 1} n_{2}^{2} \\
0 & 0 & -\dot{\gamma}_{, 2} n_{1}^{2}+\dot{\gamma}_{, 1} n_{1} n_{2} \\
0 & 0 & 0
\end{array}\right]
$$

where $n_{1}$ and $n_{2}, n_{3}=0$ are the components of the normal vector to the slip plane. The couple stress tensor can be approximated by

$$
\underset{\sim}{\boldsymbol{m}}=2 \beta \underset{\sim}{\boldsymbol{\kappa}} \simeq-2 \beta\left(\operatorname{curl}{\underset{\sim}{\boldsymbol{e}}}^{p}\right)^{T}
$$

so that

$$
(\operatorname{div} \underset{\sim}{\boldsymbol{m}})_{3} \simeq-2 \beta\left(2 \gamma_{, 12} n_{1} n_{2}-\gamma_{, 11} n_{2}^{2}-\gamma_{, 22} n_{1}^{2}\right)
$$

and

$$
x=-\frac{1}{2}(\operatorname{div} \underset{\sim}{\boldsymbol{m}})_{3} \simeq \beta\left(2 \gamma_{, 12} n_{1} n_{2}-\gamma_{, 11} n_{2}^{2}-\gamma_{, 22} n_{1}^{2}\right)
$$

This expression, when inserted into (27), gives a yield condition involving the second derivative of the amount of slip in the spirit of the model proposed by [26].

\section{Generalized kinematic hardening modulus}

The material parameter $\beta$ appearing in (34) can be regarded as a generalized kinematic hardening modulus, with the physical dimension MPa.mm². It has been evaluated by Kröner as the bending stiffness of a crystal element containing a rectangular array of edge dislocations. The existence of couple stresses in a dislocated 
crystal was put forward by Kröner [3] by considering the residual stress field after bending and torsion of single crystals. Kröner derived the expression of a material constant linking a couple-stress component to the corresponding lattice curvature tensor component, in the special case depicted in figure 1. This derivation can be interpreted as follows: to maintain a zero plane curvature in a box inside the dislocated crystal of figure 1, a torque must be applied. Conversely, in the absence of couple stresses acting on the volume element, lattice planes are curved due to the excess of dislocations of a definite sign. The characteristic length which goes into this constant is the glide plane distance $d$ between dislocations. Using the present notations, Kröner's relation reads:

$$
m_{31}=\frac{\pi^{2}}{6} \frac{\mu}{1-\nu} d^{2} \kappa_{31}
$$

corresponding to the following value of the parameter $\beta$ from (33):

$$
\beta=\frac{\pi^{2}}{12} \frac{\mu}{1-\nu} d^{2}=\frac{\pi^{2}}{12} \frac{\mu}{1-\nu} \frac{1}{\rho}
$$

where $\mu$ and $\nu$ respectively are the shear modulus and Poisson ratio of the crystal, and $d$ the distance between dislocations estimated as the inverse of the square root of the dislocation density $\rho$ of geometrically necessary dislocations.

The presence of non-homogeneous couple-stresses induces the building up of skew-symmetric force stresses as noticed in [27] following the balance equation (5).

Another interpretation of parameter $\beta$ has been given in the references $[12,13]$ considering a continuous distribution of screw dislocations bowing in a channel undergoing single slip between hard elastic layers, see figure 2. The slip plane is assumed to be perpendicular to the interfaces. During shear in a direction perpendicular to the interfaces, the dislocations continuously bow out in the channel and induce a pile-up of edge parts at the interfaces. It has been shown in $[12,13]$ that the Cosserat model (together with other strain gradient plasticity models) can capture the non-homogeneous distribution of plastic slip in the channel. The Cosserat model has been identified from the dislocation model, with the estimation

$$
\beta=\frac{2 \mu}{\rho}
$$

Let's take for instance: $\mu=40000 \mathrm{MPa}$ and $\rho=10^{12} \mathrm{~m}^{-2}$, we get $\beta=0.08 \mathrm{~Pa} \cdot \mathrm{m}^{2}=0.08 \mathrm{MPa} \cdot \mathrm{mm}^{2}$.

Both dislocation-based models indicate that the characteristic length associated with the overall Cosserat or strain gradient plasticity models:

$$
l_{c}=\sqrt{\frac{\beta}{\mu}}
$$

should not be a constant one but should evolve with deformation. Both models provide an inverse dependence of the generalized kinematic hardening modulus on the dislocation density, that can be used in the phenomenological Cosserat or gradient theories.

\section{Relation to a statistical mechanics based dislocation model}

A statistical theory of dislocations has been developed for single slip in [28,2]. It starts from the equation of motion of individual dislocations and shows that the influence of the short range dislocation-dislocation interactions can be well described by a local back-stress that includes a so-called "non-local diffusionlike term", in the spirit of [26]. The model has been compared successfully with two-dimensional discrete dislocation dynamics (DDD) simulations in [14]. A closed set of constitutive equations was formulated and 

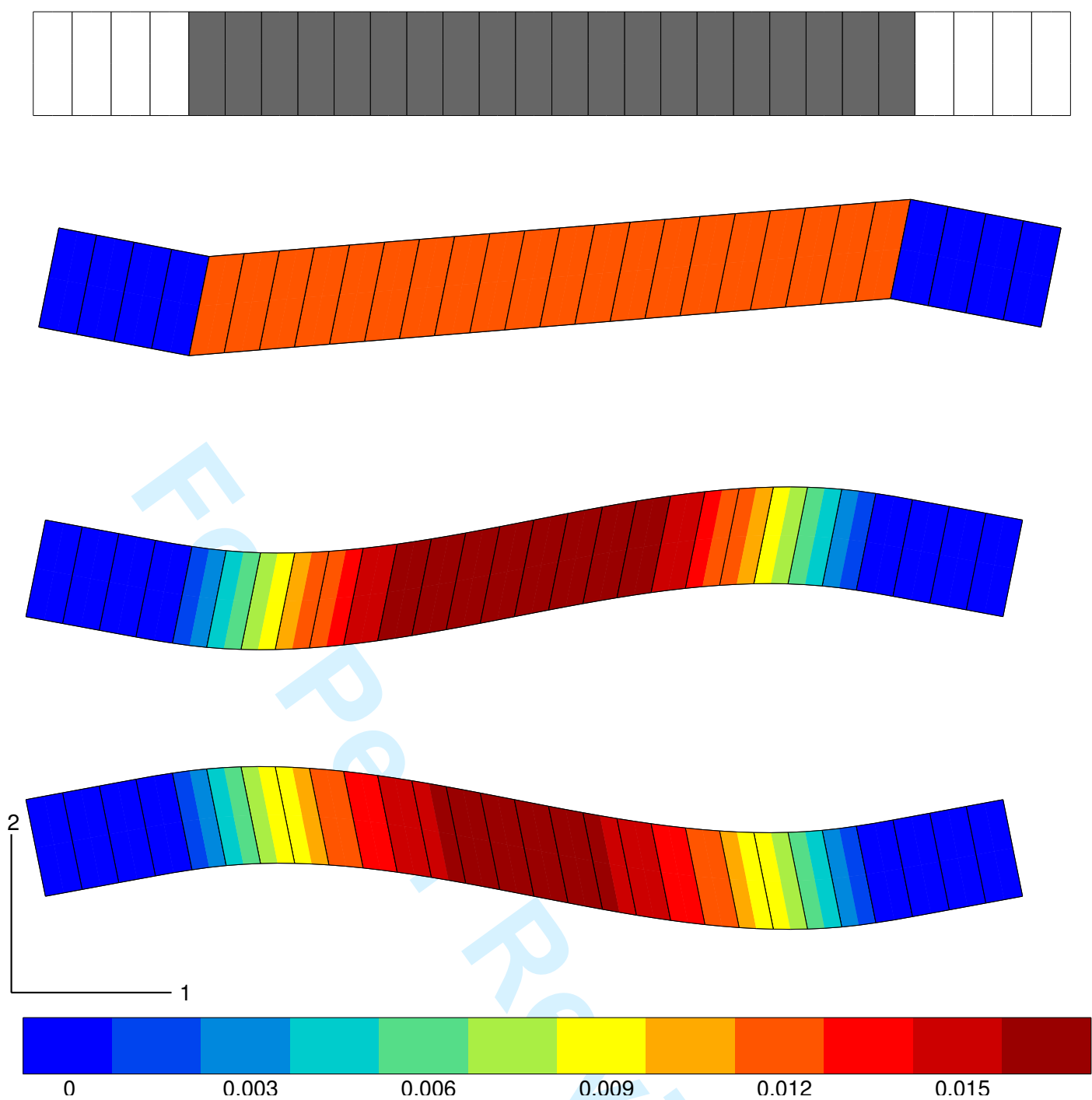

Figure 3. Periodic shearing of a two-phase laminate microstructure. From top to bottom: initial finite element mesh (hard phase in white), prescribed glide $E_{12}=0.01$ according to classical crystal plasticity, prescribed glide $E_{12}=0.01$ according to Cosserat crystal plasticity, prescribed glide $E_{12}=-0.01$ according to Cosserat crystal plasticity. The plotted field is $|\gamma|$. The material parameters are $\mu=26920 \mathrm{MPa}, \tau_{c}=10 \mathrm{MPa}, \beta=10 \mathrm{MPa} \cdot \mathrm{mm}^{2}$.

incorporated into the continuum crystal plasticity framework by these authors in the form:

$$
\begin{aligned}
& \dot{\sim}^{p}=\dot{\gamma} \frac{1}{2}(\underline{\boldsymbol{l}} \otimes \underline{\boldsymbol{n}}+\underline{\boldsymbol{n}} \otimes \underline{\boldsymbol{l}}), \quad \dot{\gamma}=\rho \underline{\boldsymbol{b}} \cdot \underline{\boldsymbol{v}} \\
& \underline{\boldsymbol{v}}=\frac{\underline{\boldsymbol{b}}}{B}(\tau-x) \\
& x=\frac{D \mu \underline{\boldsymbol{b}}}{2 \pi(1-\nu) \rho} \cdot \frac{\partial \kappa}{\partial \underline{\boldsymbol{r}}}
\end{aligned}
$$

where $B, D$ are material parameters. The Burgers vector is $\underline{\boldsymbol{b}}$. The total and excess dislocation densities $\rho=\rho^{+}+\rho^{-}, \kappa=\rho^{+}-\rho^{-}$, fulfill the following evolution partial differential equations:

$$
\dot{\rho}+\operatorname{div}(\kappa \underline{\boldsymbol{v}})=f
$$




$$
\dot{\kappa}+\operatorname{div}(\rho \underline{\boldsymbol{v}})=0
$$

where the function $f$ accounts for dislocation creation and annihilation.

In the case of single slip with $\underline{\boldsymbol{v}}=v \underline{\boldsymbol{e}}_{1}$ and $\underline{\boldsymbol{b}}=b \underline{\boldsymbol{e}}_{1}=b \underline{\boldsymbol{l}}$, the equation (59) reduces to

$$
\dot{\kappa}+\frac{1}{b} \dot{\gamma}_{, 1}=0
$$

so that the back-stress takes the form:

$$
x=-\frac{\alpha \mu}{\rho} \gamma, 11
$$

with $\alpha$ a material parameter. This relation is to be compared to (34) and (50) derived in the Cosserat model. In particular, setting $n_{1}=0$ in (50), i.e. for a slip plane normal to direction 2 , the same dependence on $\gamma_{, 11}$ is retrieved. The inverse dependence of the generalized kinematic hardening modulus with $\rho$ is in accordance with the estimations of the bending stiffness $\beta$ in section 7 , but obtained here in a more general context.

\section{Cyclic plasticity in a two-phase laminate}

The problem of the mechanical behaviour of the two-phase laminate of figure 2 initially studied in the references $[12,13]$, is reexamined here in the context of cyclic deformation and derivation of the resulting global kinematic hardening. Screw dislocations are gliding in a channel along a glide plane perpendicular to the plane parallel interfaces, under the action of a shear stress $\tau=\sigma_{12}$. They deposit edge parts along the interface inducing non-homogeneous plastic deformation in the direction 1 in the channel, and internal stresses associated with the pile-ups. The test is controlled by the prescribed mean total amount of glide $\bar{\gamma}$. The displacement field in the channel is of the form:

$$
\underline{\boldsymbol{u}}=\bar{\gamma} x_{2} \underline{\boldsymbol{e}}_{1}+u_{2}\left(x_{1}\right) \underline{\boldsymbol{e}}_{2}, \quad \underline{\boldsymbol{\Phi}}=\Phi_{3}\left(x_{1}\right) \underline{\boldsymbol{e}}_{3}
$$

with periodicity conditions for the fluctuation $u_{2}\left(x_{1}\right)$ and the micro-rotation $\Phi_{3}$ (see figure 2 for the geometry and coordinate system of the studied microstructure). The hard phase is regarded as an elastic Cosserat medium with a characteristic length much smaller than the one associated with the soft phase. This allows the transmission of the force and couple traction vectors and the continuity of displacement and micro-rotation, at the interface between hard and soft phases. In the elastic hard phase, the microrotation coincides with the material rotation. The active component of the curvature tensor inside the plastic zone is

$$
\kappa_{31}=2 \beta \Phi_{3,1}
$$

According to the balance of momentum equation $\sigma_{21,1}=0$, the stress component $\sigma_{21}$ is constant. The Schmid law (27) indicates that the stress component $\sigma_{12}=\tau_{c}$ is constant. When applied to the equation of balance of moment of momentum (5), and assuming a constant parameter $\beta$, this gives:

$$
m_{31,1}-\sigma_{12}+\sigma_{21}=0 \Longrightarrow m_{31,11}=0 \Longrightarrow \Phi_{3,111}=0
$$

which leads to a parabolic profile of lattice rotation in the channel. This is the same qualitative result as in [13] where however only the symmetric part of the stress was considered for the evaluation of Schmid law. This periodic boundary value problem has also been solved based on the finite element method, as shown in figure 3 for cyclic loading $\bar{\gamma}= \pm 0.01$. In particular the field $\gamma\left(x_{1}\right)$ is given in figure 3 . The curvature effects in the channel are clearly visible. 


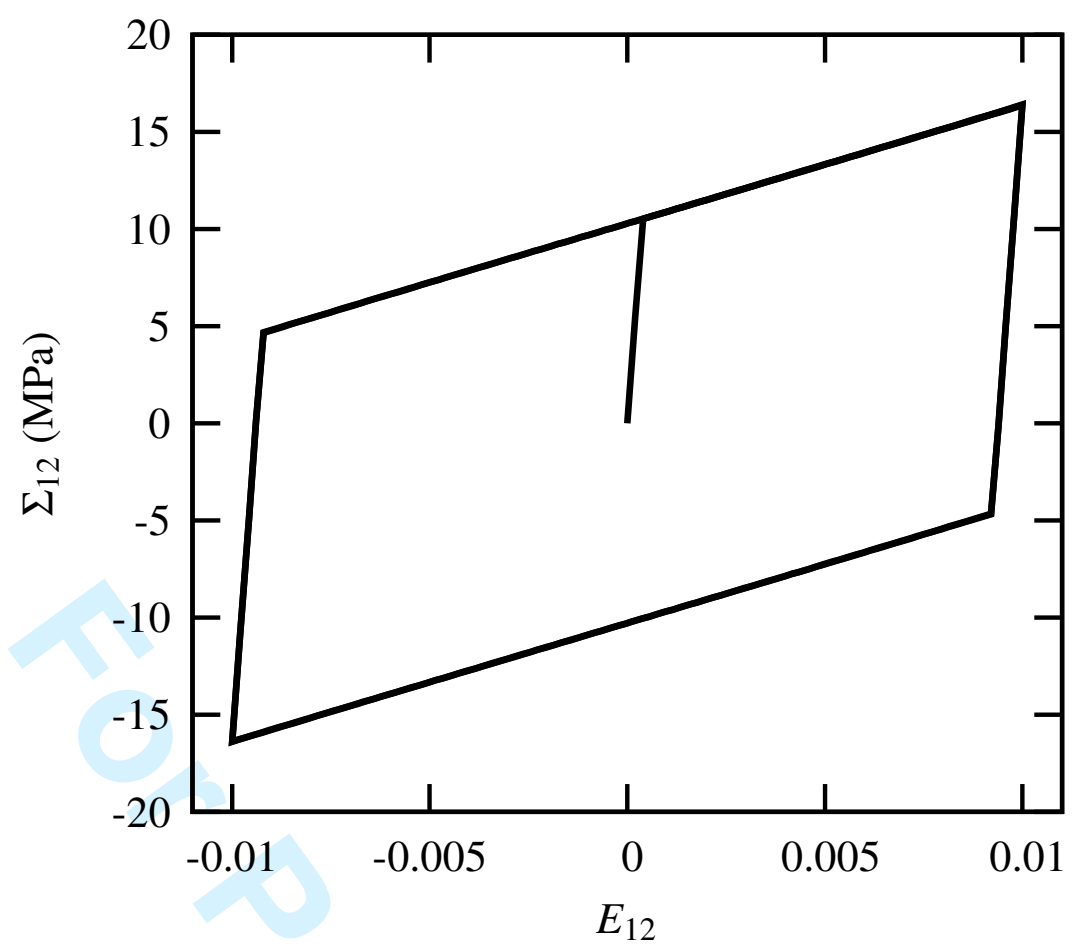

Figure 4. Mean stress-strain curve for the cycling shearing of a two-phase laminate microstructure $\left(\mu=26920 \mathrm{MPa}, \tau_{c}=10 \mathrm{MPa}\right.$, $\left.\beta=10 \mathrm{MPa} \cdot \mathrm{mm}^{2}\right)$.

The effective properties of such a laminate material can be derived from the periodic unit cell by proper averaging procedures. Such homogenization techniques have been developed for heterogeneous Cosserat media in [29]. The nature of the effective medium depends on the ratio of the Cosserat characteristic lengths and on the size of constituents. We use the averaging relations obtained for an effective Cosserat medium:

$$
\Sigma_{12}=<\sigma_{12}>=\frac{1}{V} \int_{V} \sigma_{12} d V, \quad E_{12}=<u_{1,2}>=\frac{1}{V} \int_{V} u_{1,2} d V=\bar{\gamma}
$$

The stress-strain loop obtained for $\bar{\gamma}= \pm 0.01$ is shown in figure 4, revealing effective linear kinematic hardening, induced by the internal stresses in the channel. The observed effective kinematic hardening modulus is found to be directly proportional to the parameter $\beta$.

The generated linear kinematic hardening is similar to the one derived by Tanaka and Mura in the case of a regular distribution of pile-ups in a grain [30], as analyzed in [13]. The kinematic hardening modulus scales with the inverse of the channel width $s$.

\section{Conclusions}

Two main links between available phenomenological generalized continuum crystal plasticity models and the statistical approach to dislocation dynamics have been found:

- In the Cosserat model, the skew-symmetric part of the force stress tensor induces a local back-stress in the generalized Schmid law. This back-stress is a projection of the divergence of couple stresses. A similar contribution exists in strain gradient plasticity models involving the effect of the curl of plastic deformation. In the case of single slip, the latter form of the internal stress is identical to the contribution found in the statistical theory of dislocations of $[28,2]$.

- Dislocation models based either on square arrays of edge dislocations or on the glide of screw dislocations in a channel systematically lead to the existence of a generalized linear kinematic hardening modulus 
which is proportional to the inverse of dislocation density. This dependence can be directly implemented in the phenomenological generalized continuum model. It is also in accordance with the prediction of the statistical approach.

The analysis of the simple case of heterogeneous plastic slip in a channel shows that the internal stresses result in an effective linear classical kinematic hardening on the overall stress-strain curve. Non-linearity can be introduced when the dependence of parameter $\beta$ on dislocation density is implemented. In the given examples, the total dislocation density coincides with the GND content. In more general cases, it is conjectured that the generalized kinematic hardening modulus should depend on the GND part only.

The question arises of the limit case when $\rho$ is going to zero. The kinematic hardening modulus $\beta$ increases for decreasing $\rho$. On the other hand, the curvature is going to zero when density of dislocations in excess decreases. This may result in an indeterminate value of the couple stress according to (33). In the statistical theory of dislocations, it is not excluded that the dislocation density $\rho$ appearing in the expression of back-stress may include parts of both statistical and geometric contents. One may consider the case of a fixed dislocation density $\rho^{S}$ and a vanishing curvature, or equivalently, density $\rho^{G} \rightarrow 0$, which leads to vanishing couple stresses and associated back-stress.

For single slip, most strain gradient plasticity models coincide in predicting a back-stress related to the second derivative of slip in the slip direction. Extensions to the multislip case are currently mainly heuristic and the phenomenological approach based on generalized continua can help formulating them in a consistent manner. Formulations that explicitly introduce the gradient of individual slip or dislocation densities $[15,16,31]$ share the drawback that (dis-) continuity conditions at grain boundaries cannot be formulated unambiguously because the variables $\gamma^{\alpha}, \rho^{\alpha}$ are defined in each grain only up to a crystal symmetry [32]. In contrast, plausible extensions can be proposed based on the rotational part of the full plastic deformation, i.e. the full dislocation density tensor in $[9,17]$, or on the full lattice torsion-curvature tensor $[1,21]$. These extensions have also the advantage that the number of degrees of freedom does not increase when the number of slip systems increases. In the Cosserat model for instance, the number of degrees of freedom remains equal to 3 , the three components of the lattice rotation vector, irrespective of the crystallography of the studied system. The specific crystallographic structure of the studied materials enters the model via the number of internal variables, for instance the number of slip variables $\gamma^{s}$ which depends on the number of slip systems.

\section{References}

[1] S. Forest, G. Cailletaud, and R. Sievert. A Cosserat theory for elastoviscoplastic single crystals at finite deformation. Archives of Mechanics, 49(4):705-736, 1997.

[2] I. Groma, F.F. Csikor, and M. Zaiser. Spatial correlations and higher-order gradient terms in a continuum description of dislocation dynamics. Acta Materialia, 51:1271-1281, 2003.

[3] E. Kröner. On the physical reality of torque stresses in continuum mechanics. Int. J. Engng. Sci., 1:261-278, 1963.

[4] Mura, T. Continuous distribution of dislocations and the mathematical theory of plasticity. Phys. stat. sol., 10:447-453, 1965.

[5] N.A. Fleck and J.W. Hutchinson. Strain gradient plasticity. Adv. Appl. Mech., 33:295-361, 1997.

[6] J.F. Nye. Some geometrical relations in dislocated crystals. Acta Metall., 1:153-162, 1953.

[7] P. Steinmann. Views on multiplicative elastoplasticity and the continuum theory of dislocations. International Journal of Engineering Science, 34:1717-1735, 1996.

[8] S. Forest, R. Sievert, and E.C. Aifantis. Strain gradient crystal plasticity : Thermomechanical formulations and applications. Journal of the Mechanical Behavior of Materials, 13:219-232, 2002.

[9] M.E. Gurtin. A gradient theory of single-crystal viscoplasticity that accounts for geometrically necessary dislocations. Journal of the Mechanics and Physics of Solids, 50:5-32, 2002.

[10] H. Zorski. Statistical theory of dislocations. Int. J. Solids Structures, 4:959-974, 1968.

[11] M. Zaiser, M.C. Miguel, and I. Groma. Statistical dynamics of dislocation systems: The influence of dislocation-dislocation correlations. Phys. Rev. B, 64:224102, 2001.

[12] R. Sedláček and S. Forest. Non-local plasticity at microscale : A dislocation-based model and a Cosserat model. physica status solidi (b), 221:583-596, 2000 .

[13] S. Forest and R. Sedláček. Plastic slip distribution in two-phase laminate microstructures: Dislocation-based vs. generalizedcontinuum approaches. Philosophical Magazine A, 83:245-276, 2003.

[14] S. Yefimov, I. Groma, and E. Van der Giessen. A comparison of a statistical-mechanics based plasticity model with discrete dislocation plasticity calculations. J. Mech. Phys. Solids, 52:279-300, 2004.

[15] S. Yefimov and E. Van der Giessen. Multiple slip in a strain-gradient plasticity model motivated by a statistical-mechanics description of dislocations. International Journal of Solids and Structures, 42:3375-3394, 2005.

[16] C.J. Bayley, W.A.M. Brekelmans, and M.G.D. Geers. A comparison of dislocation induced back stress formulations in strain gradient crystal plasticity. International Journal of Solids and Structures, 43:7268-7286, 2006.

[17] E.M. Viatkina, W.A.M. Brekelmans, and M.G.D. Geers. Modelling of the internal stress in dislocation cell structures. European Journal of Mechanics A/solids, 26:982-998, 2007. 
[18] P. Cermelli and M.E. Gurtin. On the characterization of geometrically necessary dislocations in finite plasticity. Journal of the Mechanics and Physics of Solids, 49:1539-1568, 2001.

[19] B. Svendsen. Continuum thermodynamic models for crystal plasticity including the effects of geometrically-necessary dislocations. J. Mech. Phys. Solids, 50:1297-1329, 2002.

[20] S. Forest and R. Sievert. Elastoviscoplastic constitutive frameworks for generalized continua. Acta Mechanica, 160:71-111, 2003.

[21] S. Forest, F. Barbe, and G. Cailletaud. Cosserat modelling of size effects in the mechanical behaviour of polycrystals and multiphase materials. International Journal of Solids and Structures, 37:7105-7126, 2000

[22] R. de Borst. Simulation of strain localization: a reappraisal of the Cosserat continuum. Engng Computations, 8:317-332, 1991.

[23] C. Teodosiu. Large plastic deformation of crystalline aggregates. CISM Courses and Lectures No. 376, Udine, Springer Verlag, Berlin, 1997.

[24] W.T. Koiter. Couple-stresses in the theory of elasticity. i and ii. Proc. K. Ned. Akad. Wet., B67:17-44, 1963.

[25] H. Gao, Y. Huang, W.D. Nix, and J.W. Hutchinson. Mechanism-based strain gradient plasticity - i. theory. J. Mech. Phys. Solids, 47:1239-1263, 1999

[26] E.C. Aifantis. The physics of plastic deformation. International Journal of Plasticity, 3:211-248, 1987.

[27] F. Hehl and E. Kröner. Zum Materialgesetz eines elastischen Mediums mit Momentenspannungen. Z. Naturforschg., 20a:336-350, 1958.

[28] I. Groma. Link between the microscopic and mesoscopic length-scale description of the collective behaviour of dislocations. Phys. Rev. B, 56:5807-5813, 1997.

[29] S. Forest, F. Pradel, and K. Sab. Asymptotic analysis of heterogeneous Cosserat media. International Journal of Solids and Structures, 38:4585-4608, 2001.

[30] K. Tanaka and T. Mura. A dislocation model for fatigue crack initiation. Journal of Applied Mechanics, 48:97-103, 1981.

[31] C.J. Bayley, W.A.M. Brekelmans, and M.G.D. Geers. A three-dimensional dislocation field crystal plasticity approach applied to miniaturized structures. Philosophical Magazine, 87:1361-1378, 2007.

[32] S. Forest. The micromorphic approach for gradient elasticity, viscoplasticity and damage. ASCE Journal of Engineering Mechanics, in press, 2008. 


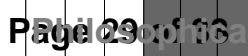


$2 0 \longdiv { 1 }$

osragleical diagazine \& Philosophical Magazine

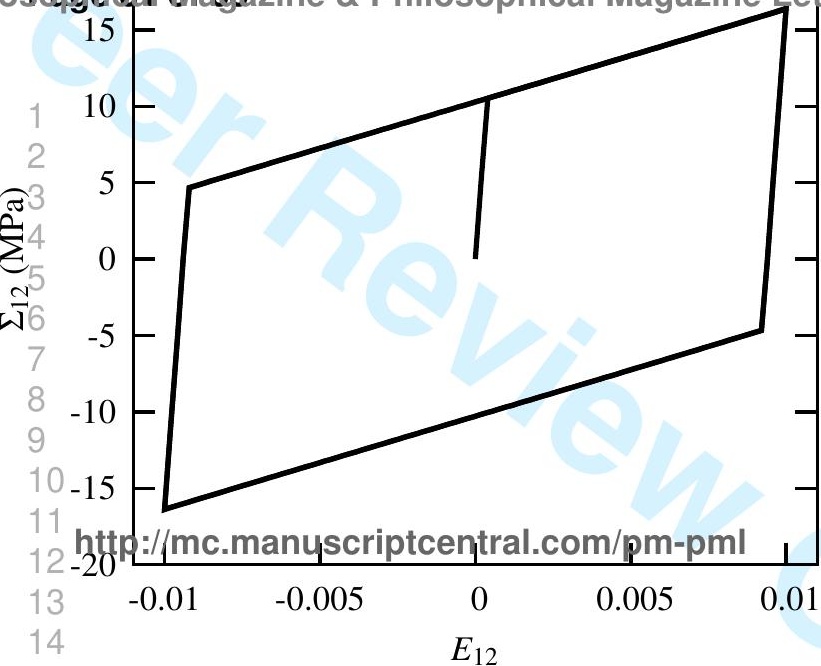




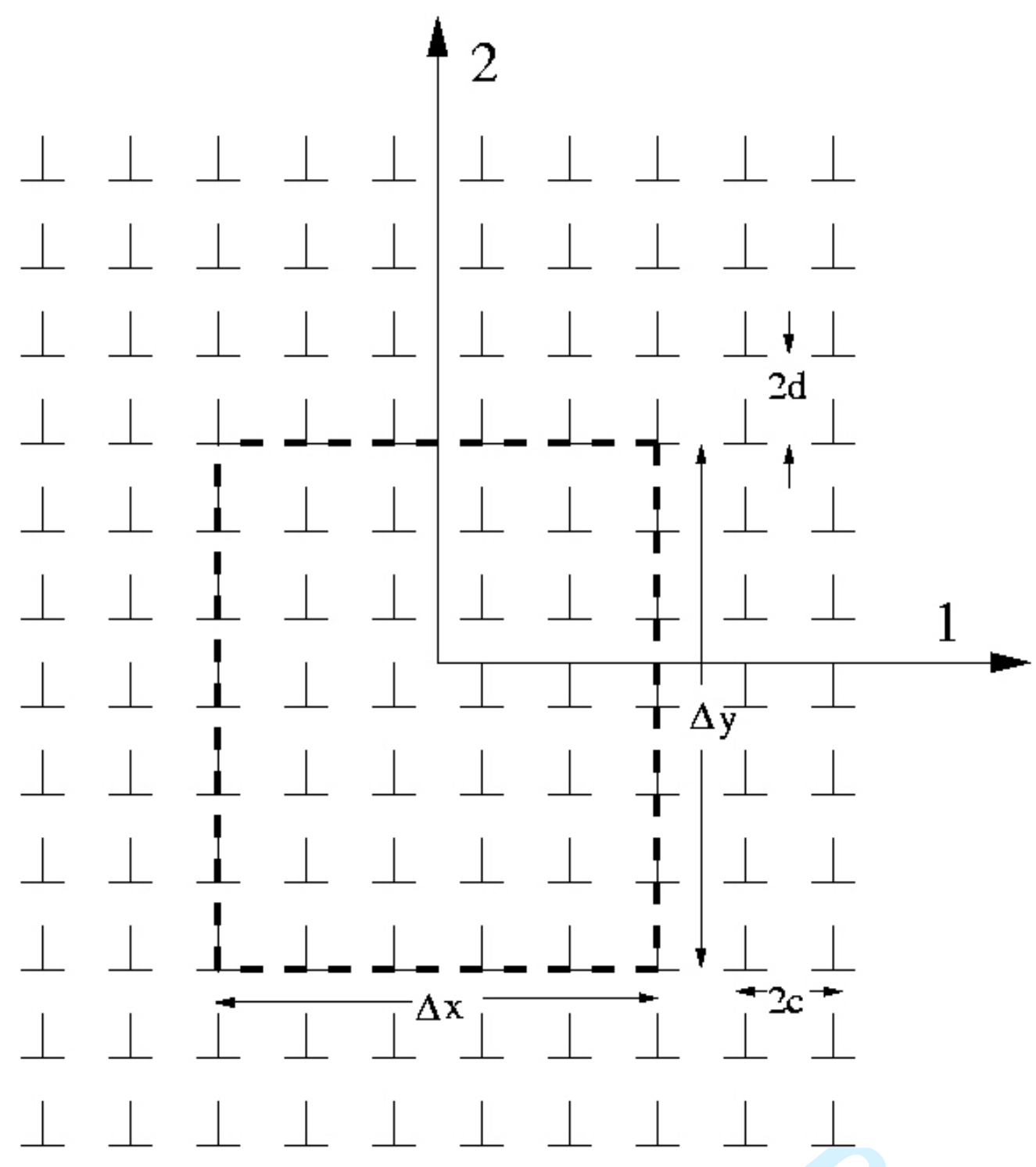


Page 33 of 33 Philosophical Magazine \& Philosophical Magazine Letters

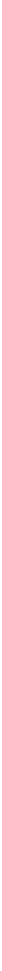

